\title{
27. IGNEOUS GEOCHEMISTRY AND PETROGENESIS OF THE IZU-BONIN FOREARC BASIN ${ }^{1}$
}

\author{
Rex N. Taylor, ${ }^{2}$ Henriette Lapierre, ${ }^{3}$ Phillipe Vidal, ${ }^{4}$ Robert W. Nesbitt, ${ }^{2}$ and Ian W. Croudace ${ }^{2}$
}

\begin{abstract}
Major element, trace element, and radiogenic isotope compositions of samples collected from Ocean Drilling Program Leg 126 in the Izu-Bonin forearc basin are presented. Lavas from the center of the basin (Site 793) are high-MgO, low-Ti, two-pyroxene basaltic andesites, and represent the products of synrift volcanism in the forearc region. These synrift lavas share many of the geochemical and petrographic characteristics of boninites. In terms of their element abundances, ratios, and isotope systematics they are intermediate between low-Ti arc tholeiites from the active arc and boninites of the outer-arc high. These features suggest a systematic geochemical gradation between volcanics related to trench distance and a variably depleted source.

A basement high drilled on the western flank of the basin (Site 792) comprises a series of plagioclase-rich two-pyroxene andesites with calc-alkaline affinities. These lavas are similar to calc-alkaline volcanics from Japan, but have lower contents of $\mathrm{Ti}, \mathrm{Zr}$, and low-field-strength elements (LFSE).

Lavas from Site 793 show inter-element variations between $\mathrm{Zr}$, $\mathrm{Ti}, \mathrm{Sr}, \mathrm{Ni}$, and $\mathrm{Cr}$ that are consistent with those predicted during crystallization and melting processes. In comparison, concentrations of P, Y, LFSE, and the rare-earth elements (REE) are anomalous. These elements have been redistributed within the lava pile, concentrating particularly in sections of massive and pillowed flows. Relative movement of these two-element groupings can be related to the alteration of interstitial basaltic andesite glass to a clay mineral assemblage by a post-eruptive process. Fluid-rock interaction has produced similar effects in the basement lavas of Site 792. In this sequence, andesites and dacites have undergone a volume change related to silica mobility. As a result of this process, some lithologies have the major element characteristics of basaltic andesite and rhyolite, but can be related to andesitic or dacitic precursors by silica removal or addition.
\end{abstract}

\section{INTRODUCTION}

The Izu-Bonin arc-trench system is the product of about $50 \mathrm{Ma}$ of Pacific lithosphere subduction. Magmatism during this time has periodically shifted from forearc to arc and backarc during arc-chain and rift-basin development, creating distinct volcanotectonic units. One of the aims of Leg 126 was to investigate the origin, timing, and products of the Izu-Bonin forearc rift-basin. The forearc basin is $40-80 \mathrm{~km}$ across and can be traced as a basement feature for $2000 \mathrm{~km}$ from south of Japan to east of Guam aside the Izu, Volcano, and Mariana arcs. Drilling results from Leg 126 have shown that this basin initiated during the Oligocene (about 30-32 Ma) by the stretching of formerly contiguous outer-arc high and remnant arc terranes of Eocene and early Oligocene age (Leg 126 Shipboard Scientific Party, $1989 \mathrm{a} ; 1989 \mathrm{~b})$. The basin was rapidly filled by volcaniclastic material derived from the uplifted blocks (Taylor, Fujioka, et al., 1990), which produced the 1-4-km-thick sedimentary cover.

To investigate the nature of the previously unsampled basement flooring the Izu-Bonin forearc basin, two deep holes were drilled though the sedimentary fill (Figs. 1 and 2). The first, Hole 793B, penetrated $1404 \mathrm{~m}$ of sediment in the center of the basin before entering volcanic basement. In addition to basement, igneous material was recovered from a Neogene sill in Hole 793B and as clasts within the coarser volcaniclastic units. The second, Hole 792E, was located on a frontal-arc high in the west of the forearc basin (Figs. 1 and 2) and reached basement at 804 mbsf. This study details the petrology and geochemistry of the igneous units recovered from the forearc, and discusses their petrogenesis and significance within the framework of intraoceanic arc evolution.

\footnotetext{
'Taylor, B., Fujioka, K., et al., 1992. Proc. ODP, Sci. Results, 126: College Station, TX (Ocean Drilling Program).

${ }^{2}$ Department of Geology, The University of Southampton, Southampton, SO9 5NH, United Kingdom.

${ }^{3}$ URA-CNRS 1366, 69, Université Joseph Fourier, Institute Dolomieu, 15 Rue Maurice Gignoux, 38031 Grenoble Cedex, France.

${ }^{4}$ Unité Associée no. 10, CNRS et Université Blaise Pascal, Clermont-Ferrand, France.
}

\section{SITE 793}

\section{Rift Volcanism in the Forearc}

Constraints from multichannel seismic surveys and Leg 126 drilling have shown that the Izu-Bonin forearc basin was initiated during the middle to late Oligocene (Taylor and Mitchell, this volume). Rifting of the Eocene basement, presently exposed on the Bonin Islands and recovered at Site 786 (ODP Leg 125; Fryer, Pearce, Stokking, et al., 1990), produced a series of half-graben structures on the eastern flank of the basin. Oligocene turbidites rapidly filled these structures and lapped onto footwall basement of the outer-arc high. Late Oligocene-Miocene strata show a decline in sedimentation rates with deposition of more calcareous and fine-grained facies. Renewed arc activity between $6 \mathrm{Ma}$ and the present is recorded in the Pliocene/ Quaternary strata as mixtures of scoriaceous and pumiceous sands and gravels. Growth of the currently active volcanic edifices is also displayed as a downwarp of seismic reflectors toward the arc volcanoes. This is caused in part by the loading effect of the developing volcanic mass on the thin arc crust (Taylor et al., 1990).

\section{Volcanic Stratigraphy}

Beneath the forearc basin sediments, Hole 793B penetrated $279 \mathrm{~m}$ of volcanic basement, consisting of intercalated volcanic breccias $(65 \%)$, pillowed flows $(15 \%)$, and massive flows (20\%), as illustrated in Figure 3. The volcanic breccias are predominantly monolithic, containing clasts of pillow and massive flow of similar compositions to the adjacent lavas. The matrix to these clasts consists of relict glass shards, fine lava debris, and phenocrysts. Clast sorting and heterolithic fragments within the uppermost breccia (Unit 1) indicate local reworking of the volcaniclastic material.

The basement sequence represents the product of a submarinerift volcano. The high proportion of volcanic breccias can be related to synvolcanic half-graben faulting, which would provide a number of unstable scarps along which volcanic debris and lava flows could accumulate. The Unit 1 breccia represents a decline in volcanic activity and a change from locally derived clastic 


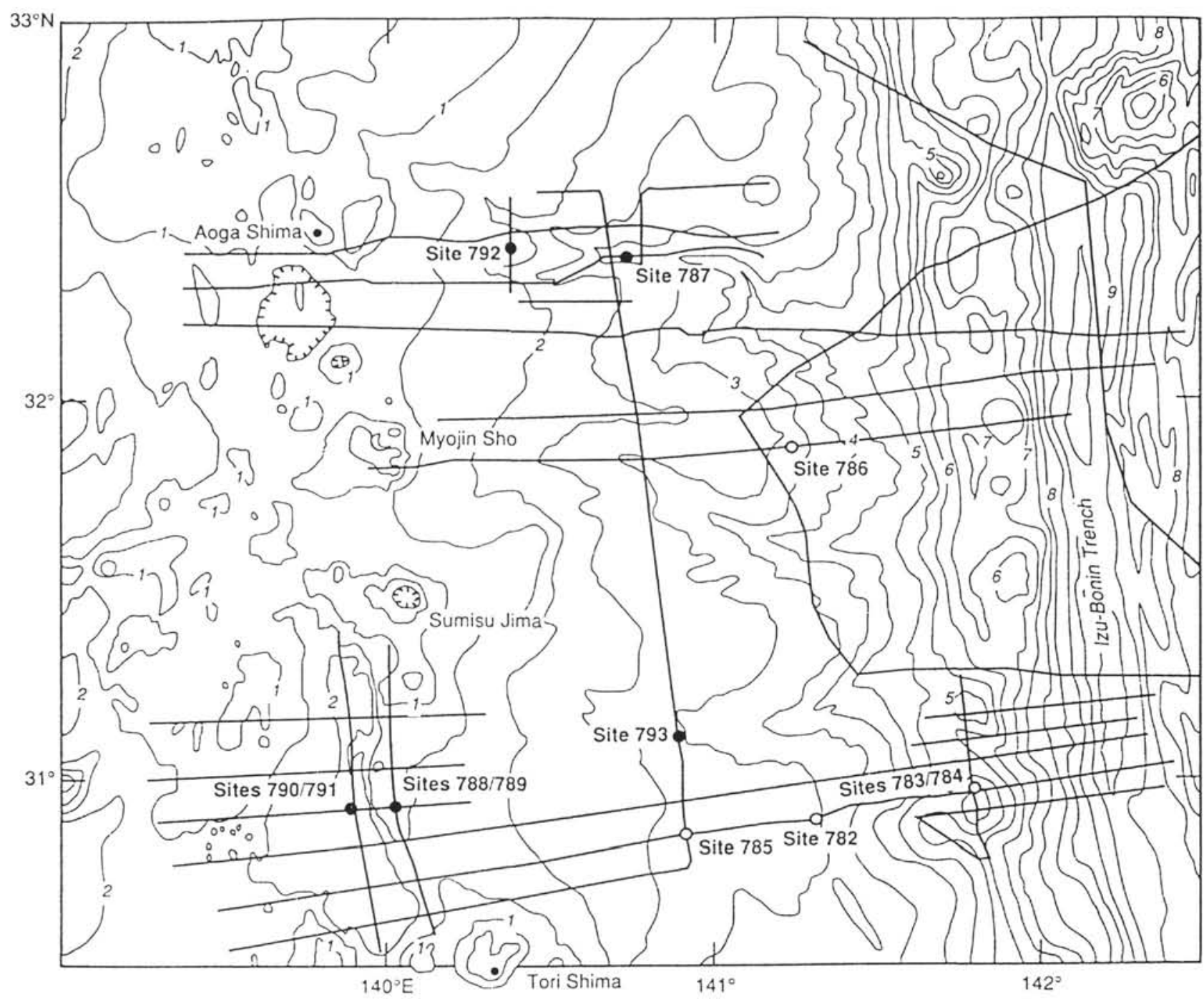

Figure 1. Bathymetric map of the Izu-Bonin arc-trench system between $30.5^{\circ}$ and $33^{\circ} \mathrm{N}$. The locations of sites drilled on Legs 125 (open circles) and 126 (solid circles) are shown on the map as are the locations of site survey multichannel seismic lines. Contour interval $=500 \mathrm{~m}$ (numbers on contour lines indicate depth in km). From Taylor, Fujioka, et al. (1990).

deposition into the more distal, coarse-clastic sedimentation during further basinal stretching.

\section{Petrography}

The transition from sedimentary sequence to basement is marked by a change from volcanogenic conglomerates to a heterolithic volcanic breccia. The most apparent distinction between these units in the recovered material was the presence of highly plagioclase-phyric andesites in the sediments and pyroxene-phyric lavas in the breccia. This distinction was amplified by the petrographic study of clast material in the conglomerates and breccia. Figure 3 shows the change in phenocryst assemblage between cover and basement. The clasts in sedimentary Unit V have a plagioclaseclinopyroxene-orthopyroxene-magnetite (plag-cpx-opx-mgte) crystallization assemblage, similar to the calc-alkaline andesites recovered in Hole 792E (see below). In contrast, the basement volcanics show crystallization of the pyroxenes before plagioclase (ol-opx + cpx-plag) and magnetite is either absent or limited to the groundmass.
Basement lavas and breccia clasts are composed of four phenocryst assemblages (listed below in approximate abundance order) distributed randomly throughout the stratigraphy (see Fig. 3 and Table 1):

(i) $\mathrm{cpx}+\mathrm{opx}+$ olivine $(\mathrm{ol})+$ chrome spinel (porphyritic)

(ii) $\mathrm{cpx}+\mathrm{opx}$ (porphyritic)

(iii) $\mathrm{cpx}+\mathrm{opx}+$ plag (porphyritic)

(iv) $\mathrm{cpx}+$ plag (aphyric)

Type (iii) is the dominant assemblage in the stratigraphy. The mineral chemistry from Hole 793B basement is discussed in a parallel contribution (Lapierre et al., this volume). A typical texture from a Type (i) lava is shown in Plate 1, and brief details of the phenocryst minerals follow.

\section{Olivine}

Only represented by saponite/celadonite/hematite/calcite pseudomorphs after subhedral or anhedral grains, with inclusions of chrome 


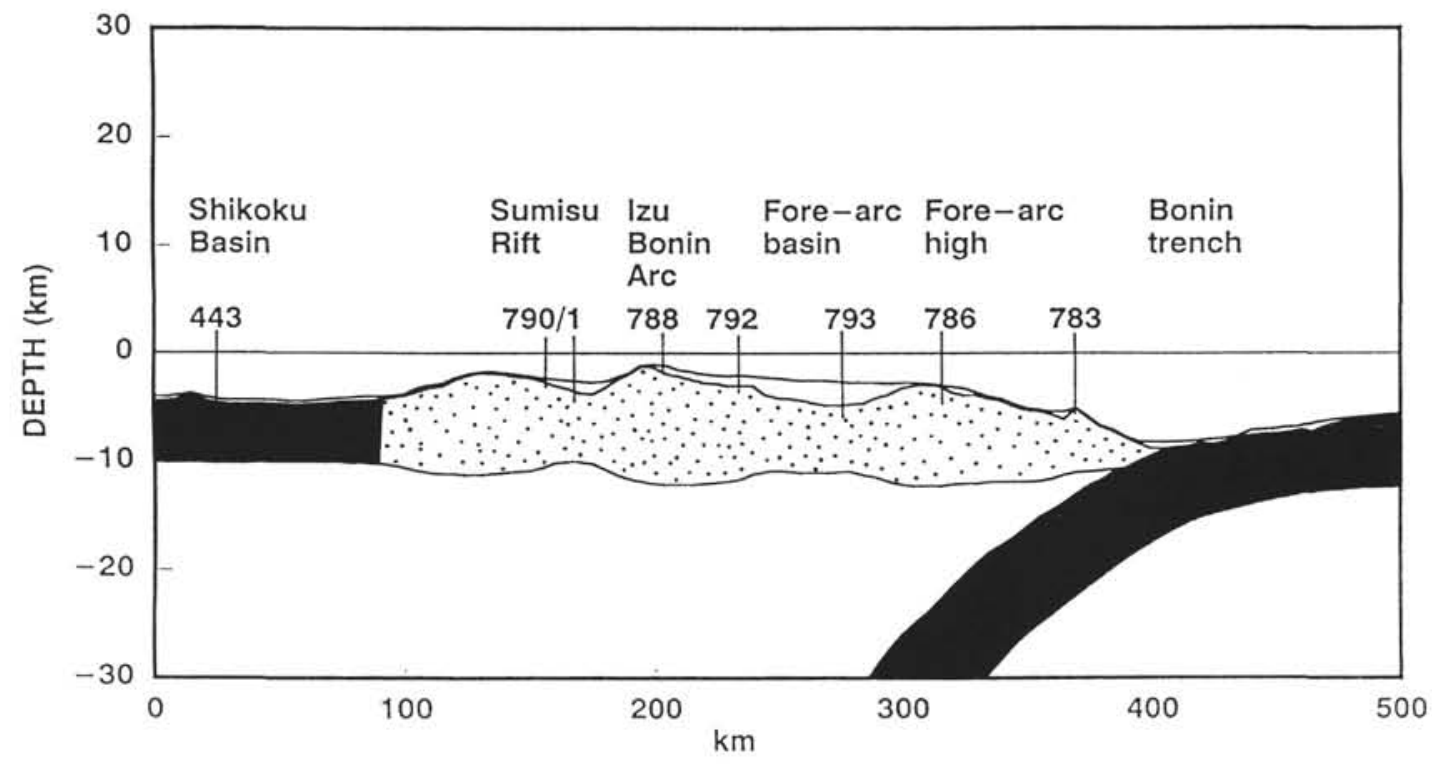

Figure 2. Schematic west-east section across the Izu-Bonin arc-trench system. Numbers refer to DSDP and ODP sites. Shaded regions are MORB-type crust; stippled regions are arc-related crust.

spinel. Unit 13 (Orange Spot flow) contains about $8 \%$ olivine pseudomorphs arranged as glomeroporphyritic clots.

\section{Clinopyroxene}

Present as fresh phenocrysts in all units of the basement. Euhedral-subhedral crystals are $1-10 \mathrm{~mm}$ in size, occasionally are twinned, and have minor compositional zoning. Compositions range from $\mathrm{En}_{39}: \mathrm{Wo}_{44}: \mathrm{Fs}_{17}$ to $\mathrm{En}_{54}: \mathrm{Wo}_{39}: \mathrm{Fs}_{7}$.

\section{Orthopyroxene}

Occurs as euhedral-subhedral phenocrysts $1-5 \mathrm{~mm}$ in size. Crystal margins and sometimes whole crystals are replaced by smectite and celadonite. Limited compositional zoning is present, but rims tend to be slightly more $\mathrm{Fe}$-rich. Compositions range from $\mathrm{En}_{82}$ to $\mathrm{En}_{88}$.

\section{Plagioclase}

Subhedral, fresh phenocrysts $0.3-2 \mathrm{~mm}$ in size. Compositions vary from $\mathrm{An}_{64}$ to $\mathrm{An}_{90}$ between phenocrysts, and from $\mathrm{An}_{60}$ to $\mathrm{An}_{86}$ in the laths and microlites of the groundmass.

\section{Geochemistry}

Major and trace element analyses were undertaken on 86 samples from the basement volcanics and lava clasts from the lowest $350 \mathrm{~m}$ of Hole 793B (Table 2). A Philips PW1400 fully automatic X-ray fluorescence spectrometer fitted with a $3 \mathrm{~kW}$ Rh anode tube was used for the analysis of all samples at the Department of Geology, University of Southampton. Major elements were obtained from fusion beads prepared from $1 \mathrm{~g}$ calcined rock powder and $5 \mathrm{~g}$ of Spectroflux 100B. Matrix effects were compensated for by using Philips' alphas (influence coefficients). Precision and accuracy are both high and nominally better than $1 \%$ (relative). Loss on ignition (LOI) was measured for each sample as the weight lost on samples between $100^{\circ} \mathrm{C}$ and $1000^{\circ} \mathrm{C}$, and is excluded from the totals reported in Tables 2 , 5 , and 7 .

Trace elements were determined on $40 \mathrm{~mm}$ diameter powder pellets pressed to 10 tonnes. Twelve drops of an $8 \% \mathrm{w} / \mathrm{v}$ aqueous solution of polyvinyl alcohol were used as a binder. Corrections for matrix effects for trace elements were made for all wavelength regions, using the Compton scatter technique (full procedural details can be found in Croudace and Gilligan, 1990). Modified corrections, necessary when crossing absorption edges, were also applied to $\mathrm{Ba}$, $\mathrm{V}$, and $\mathrm{Cr}$. Concentration calibrations were performed using carefully chosen high-quality geological reference samples (basic-intermediate composition). Precision and accuracy are generally better than $1 \%$ (relative). when the element concentrations are well above their detection limits. Detection limits are approximately $1 \mathrm{ppm}$ for $\mathrm{Zr}, \mathrm{Nb}$, $\mathrm{Y}, \mathrm{Ga}, \mathrm{Rb}, \mathrm{Sr}, \mathrm{Ni}, \mathrm{Cu}$, and $\mathrm{Zn}$ and $6 \mathrm{ppm}$ for $\mathrm{Cr}$, V, and $\mathrm{Ba}$.

Phosphorus was measured both on fusion beads and powder pellets, although the latter data are preferred because the determinations are considerably more sensitive and precise. Using powder pellets, all samples were counted by recycling the measurements six times, and counting at peak and background positions for 200 and $100 \mathrm{~s}$, respectively. Matrix corrections were applied using mass absorption coefficients calculated from major element compositions. Precision is better than $2 \%$ rel. at 100 ppm P.

\section{Major and Trace Element Systematics}

In Figure 4, major elements (recalculated anhydrous) are plotted against $\mathrm{MgO}$ for the basement volcanics. Negative correlations exist with $\mathrm{Al}_{2} \mathrm{O}_{3}$ and $\mathrm{TiO}_{2}$, whereas $\mathrm{CaO}$ and $\mathrm{Fe}_{2} \mathrm{O}_{3}$ remain roughly constant with decreasing $\mathrm{MgO}$. The plots in Figure 4 include the fields for phenocryst compositions, a bulk extract (B) composition (a composition weighted according to the average modal proportions of phenocrystal minerals present in lavas with $>7 \mathrm{wt} \% \mathrm{MgO}$ ), and a bulk-extract control line.

The major element plots demonstrate that the majority of wholerock compositions can be interrelated by the removal or addition of the bulk extract composition. This relationship is pursued further by least-squares mixing calculations (Table 3). These calculations confirm that extracting the observed modal proportions in a quantity equivalent to phenocryst abundances successfully links the wholerock compositions. As such, it is possible that groundmass or liquid compositions were similar throughout the majority of lavas, with phenocryst abundance causing whole-rock variations. This notion is supported by the lack of variation in clinopyroxene and plagioclase chemistry from lavas with widely varying $\mathrm{MgO}$ contents (Lapierre et al., this volume). 


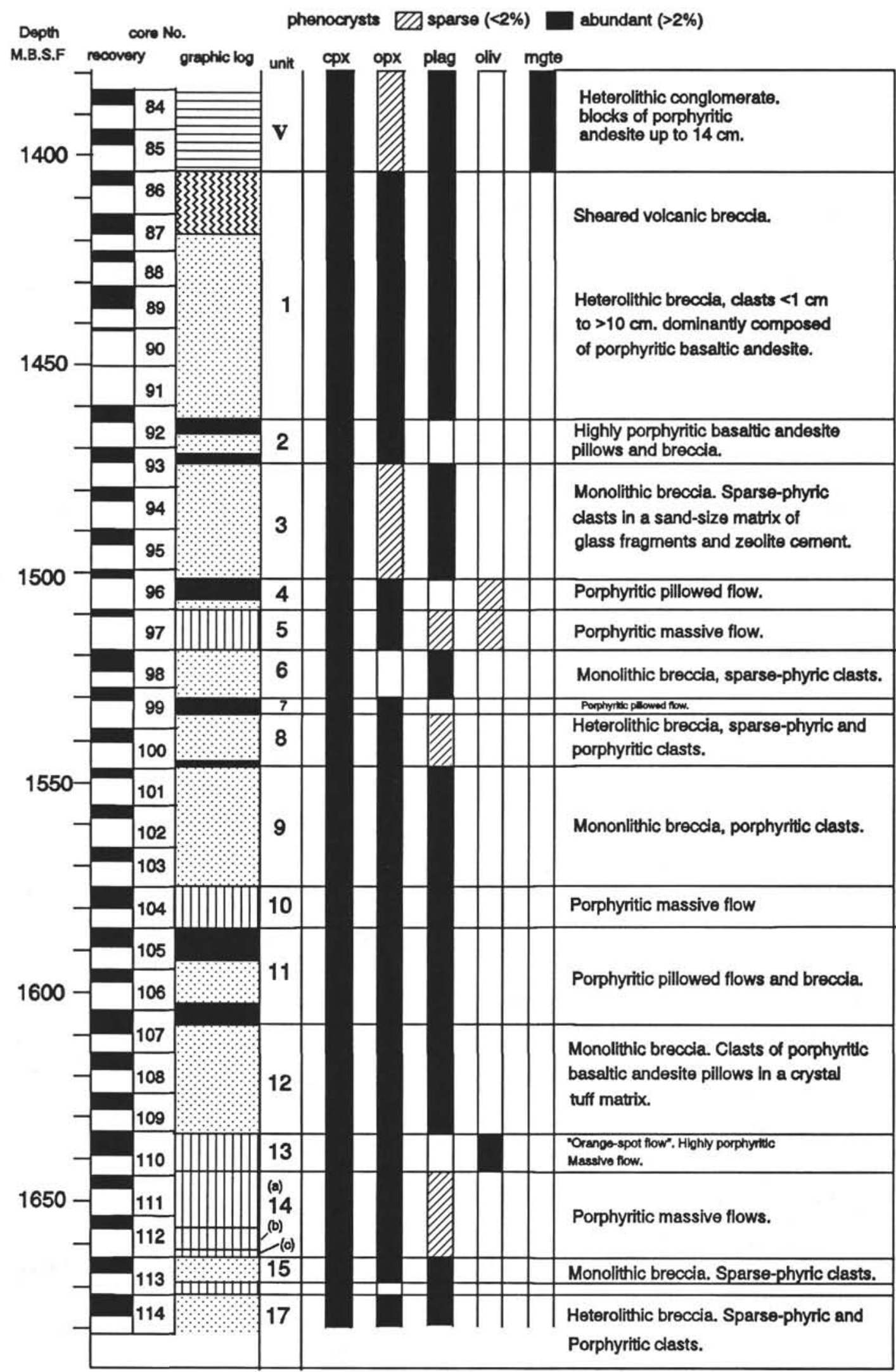

Pillowed flow W Massive flow Solcanic breccia

Figure 3. Basement stratigraphy, Hole 793B. Phenocryst assemblage throughout the stratigraphy is shown in the columns on right. Shaded $=$ abundant $(>2 \%$ modal $)$, hatched $=$ sparse $(<2 \%)$, blank $=$ absent. Abbreviations: $\mathrm{cpx}=$ clinopyroxene, opx $=$ orthopyroxene, plag $=$ plagioclase, olv $=$ olivine, and $\mathrm{mgte}=$ magnetite $/$ opaque. 
Table 1. Modal mineralogy of basement rocks, Hole 793B.

\begin{tabular}{lllrrrrrrr}
\hline $\begin{array}{c}\text { Sample/ } \\
\text { analysis }\end{array}$ & $\begin{array}{c}\text { Core, section, } \\
\text { interval (cm) }\end{array}$ & $\begin{array}{l}\text { Depth } \\
\text { (mbsf) }\end{array}$ & Unit & Cpx & Opx & Plag & Oliv & Vesic & $\begin{array}{c}\text { Petrological } \\
\text { type }\end{array}$ \\
\hline 044 & $97 \mathrm{R}-1,80$ & 1515.78 & 5 & 10.5 & 5.9 & 0.9 & 1.2 & 4.3 & (i) \\
S-19 & $110 \mathrm{R}-1,1$ & 1633.83 & 13 & 8.8 & 7.8 & 0.1 & 8.4 & 0.7 & (i) \\
030 & $92 \mathrm{R}-2,70$ & 1465.32 & 2 & 23.5 & 16.7 & 0.3 & - & 2.7 & (ii) \\
083 & $112 \mathrm{R}-2,57$ & 1661.38 & 14 & 16.6 & 12.2 & 0.5 & - & 2.9 & (ii) \\
053 & $104 \mathrm{R}-1,65$ & 1577.11 & 10 & 13.8 & 3.1 & 7.4 & - & 3.7 & (iii) \\
051 & $102 \mathrm{R}-1,128$ & 1562.51 & 9 & 1.6 & 2.5 & 3.2 & - & 6.5 & (iii) \\
058 & $105 \mathrm{R}-1$ & 1585.61 & 11 & 7.8 & 11.7 & 9.4 & - & 5.4 & (iii) \\
033 & $92 \mathrm{R}-3,55$ & 1468.50 & 2 & 6.1 & 6.7 & 3.5 & - & 2.4 & (iii) \\
028 & 89R-2,86 & 1436.13 & 1 & 7.1 & 12.4 & 1.8 & 0.7 & 3.7 & (iii) \\
071 & $109 \mathrm{R}-3,50$ & 1633.31 & 12 & 7.5 & 10.1 & 6.1 & - & 0.2 & (iii) \\
050 & 102R-1, 36 & 1558.33 & 8 & 4.0 & 1.0 & 3.6 & - & 2.4 & (iv) \\
039 & $94 \mathrm{R}-1,100$ & 1483.21 & 3 & 2.1 & 1.4 & 2.0 & - & 4.8 & (iv) \\
\hline
\end{tabular}

Notes: All analyses performed by point counting ( $>800$ counts per section). Reported values are percentages, with groundmass the residual percentage. Petrological types are defined in the text, unit numbers refer to position in stratigraphy (see Fig. 3).
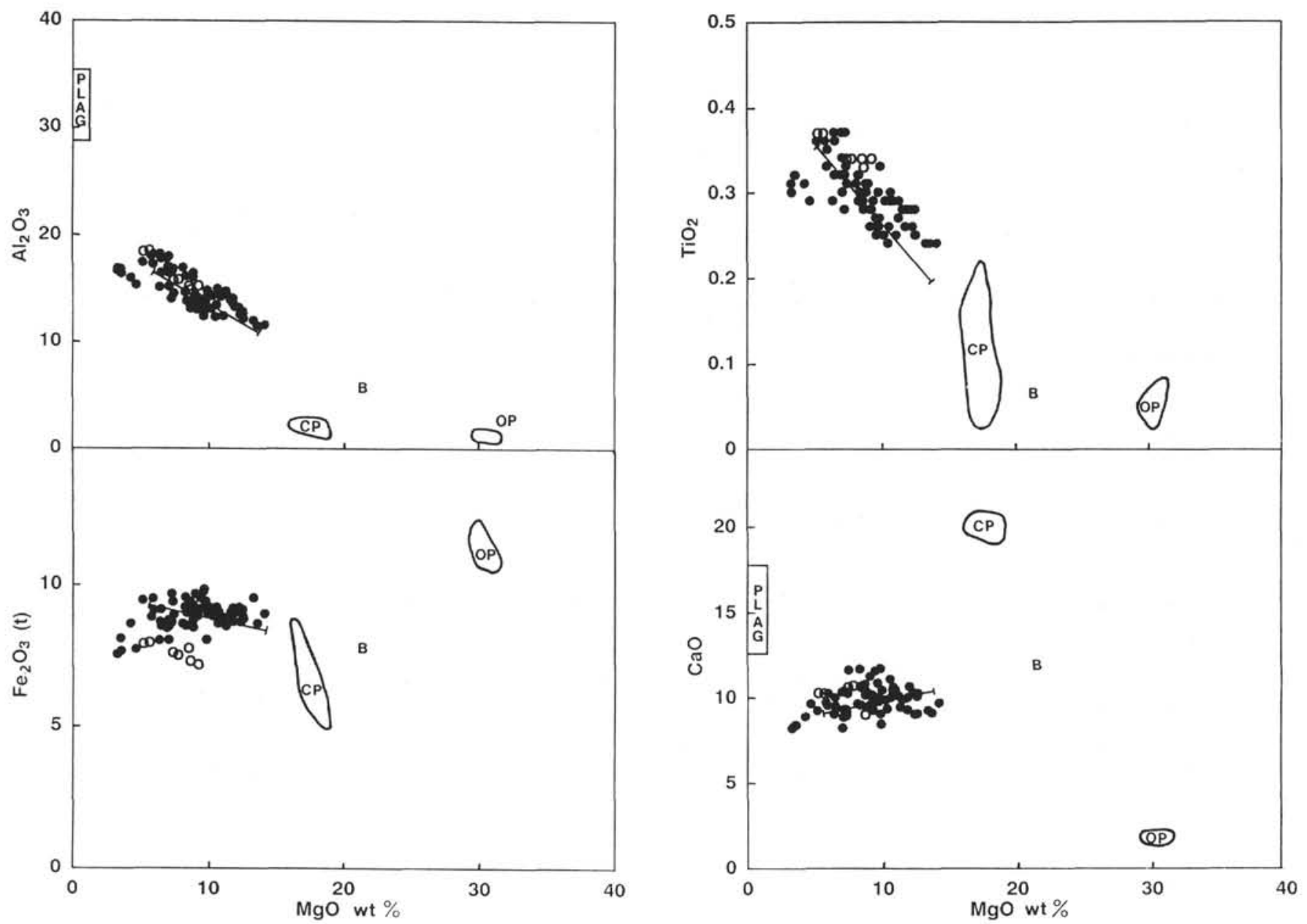

Figure 4. Major element variation with $\mathrm{MgO}$ for Hole 793B basement lavas. Open circles are Group A lavas, and solid circles are Group B lavas (see text). Fields are shown for the range of phenocryst compositions present in Sample 033 (analyses from Lapierre et al., this volume). $\mathrm{OP}=$ orthopyroxene, $\mathrm{CP}=$ clinopyroxene, and PLAG = plagioclase. Bulk extract $(\mathrm{B})$ is a composition derived from average phenocryst analyses weighted according to their modal proportions with Sample 033 . A mixing line between Group B lavas and Sample $050(5.13 \mathrm{wt} \% \mathrm{MgO})$ represents between $2 \%$ and $55 \%$ addition of bulk extract to Sample 050 .

Combining modal analyses of samples with geochemical data indicates that the proportion of individual phenocryst types changes with whole-rock chemistry. This is demonstrated in Figure 5, which shows an increase in plagioclase (as a percentage of phenocrysts present in each sample) and a decrease in orthopyroxene with declining whole-rock $\mathrm{MgO}$ content. As such, it is likely that whole-rock compositions are a partial function of magma evolution. However, phenocryst content appears to be the dominant factor in whole-rock geochemical variations.

In Figure 6, trace elements are plotted against $\mathrm{Zr}$ as a fractionation index. The samples are divided into two groups on these plots on the basis of stratigraphic and geochemical differences. The first group (Group A) comprises the upper seven samples within Unit 1. These lava clasts have higher $\mathrm{Zr}$ and lower $\mathrm{Fe}$ contents than all underlying 
Core, section

Interval

Depth (MBSF

Sample no.

Unit

Lithology

86R-1 86R-2 86R-2 87R-3 87R 88R-1 88R-1 89R-2 89R-2 89R-2 92R-1 92R-2 92R-2 92R-3 92R-3 92R-3 92R-cc 93R-1 93R-1

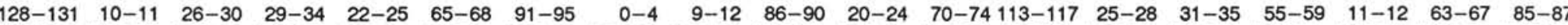

$\begin{array}{llllllllllllllllllll}1408.37 & 1409.50 & 1410.11 & 1419.77 & 1420.60 & 1425.50 & 1426.61 & 1434.44 & 1434.60 & 1436.13 & 1460.62 & 1465.32 & 1466.34 & 1467.79 & 1467.96 & 1468.50 & 1469.51 & 1471.80 & 1472.47\end{array}$

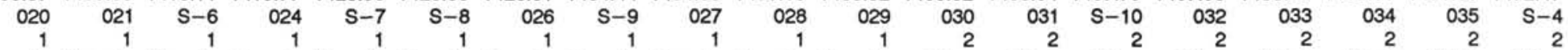

Breccia Breccia Breccia Breccia Breccia Breccia Breccia Breccia Breccia Breccia Breccia Pillow Pillow Pillow Pillow Pillow Pillow Pillow Pillow

Major elements (wt\%)

\begin{tabular}{|c|c|c|c|c|c|c|c|c|c|c|c|c|c|c|c|c|c|c|c|}
\hline $\mathrm{SiO} 2$ & 54.97 & 56.23 & 55.33 & 57.82 & 55.03 & 53.89 & 54.40 & 54.98 & 54.81 & 54.59 & 54.77 & 53.46 & 53.52 & 54.11 & 53.62 & 54.19 & 54.54 & 53.03 & 52.63 \\
\hline $\mathrm{TiO} 2$ & 0.34 & 0.34 & 0.34 & 0.33 & 0.34 & 0.37 & 0.37 & 0.28 & 0.32 & 0.29 & 0.26 & 0.25 & 0.24 & 0.30 & 0.28 & 0.31 & 0.32 & 0.28 & 0.28 \\
\hline $\mathrm{Al} 2 \mathrm{O} 3$ & 15.84 & 15.24 & 15.79 & 14.87 & 15.21 & 18.46 & 18.58 & 13.62 & 16.40 & 14.21 & 12.43 & 12.07 & 11.46 & 13.87 & 14.05 & 15.97 & 16.45 & 13.20 & 13.15 \\
\hline $\mathrm{Fe} 2 \mathrm{O}_{3}$ * & 7.50 & 7.16 & 7.59 & 7.29 & 7.74 & 7.91 & 7.95 & 9.09 & 9.08 & 8.84 & 8.64 & 8.74 & 8.93 & 8.44 & 8.84 & 8.70 & 8.64 & 8.96 & 9.16 \\
\hline $\mathrm{MnO}$ & 0.15 & 0.20 & 0.14 & 0.16 & 0.16 & 0.12 & 0.13 & 0.14 & 0.13 & 0.15 & 0.16 & 0.17 & 0.18 & 0.15 & 0.15 & 0.15 & 0.13 & 0.19 & 0.19 \\
\hline $\mathrm{MgO}$ & 7.77 & 9.26 & 7.40 & 8.68 & 8.55 & 5.21 & 5.65 & 9.23 & 6.47 & 10.27 & 12.32 & 12.56 & 14.12 & 8.86 & 9.17 & 8.82 & 7.26 & 11.92 & 12.20 \\
\hline $\mathrm{CaO}$ & 10.71 & 9.78 & 10.63 & 9.04 & 10.61 & 10.29 & 10.27 & 9.23 & 9.46 & 9.30 & 9.00 & 10.21 & 9.65 & 10.24 & 10.12 & 9.44 & 9.10 & 10.61 & 10.28 \\
\hline $\mathrm{Na} 2 \mathrm{O}$ & 1.65 & 1.48 & 1.73 & 1.66 & 1.81 & 2.23 & 2.24 & 1.78 & 2.15 & 1.75 & 1.57 & 1.78 & 1.45 & 2.30 & 2.25 & 2.03 & 2.31 & 1.59 & 1.57 \\
\hline K2O & 0.93 & 0.43 & 1.12 & 0.82 & 0.66 & 0.92 & 0.91 & 1.09 & 1.28 & 0.56 & 0.46 & 0.58 & 0.18 & 0.88 & 1.03 & 0.42 & 0.89 & 0.20 & 0.26 \\
\hline $\mathrm{P} 2 \mathrm{O} 5$ & 0.01 & 0.03 & 0.02 & 0.03 & 0.03 & 0.05 & 0.03 & 0.02 & 0.02 & 0.02 & 0.01 & 0.05 & 0.02 & 0.36 & 0.05 & 0.02 & 0.03 & 0.03 & 0.03 \\
\hline (LOI) & 2.44 & 3.07 & 1.18 & 4.47 & 1.69 & 1.45 & 2.18 & 1.92 & 2.05 & 1.82 & 2.51 & 1.05 & 1.19 & 1.35 & 1.15 & 1.90 & 2.33 & 2.79 & 1.64 \\
\hline Total & 99.87 & 100.15 & 100.09 & 100.70 & 100.14 & 99.45 & 100.53 & 99.46 & 100.12 & 99.98 & 99.62 & 99.87 & 99.75 & 99.51 & 99.56 & 100.05 & 99.67 & 100.01 & 99.75 \\
\hline
\end{tabular}

Trace elements (ppm)

\begin{tabular}{|c|c|c|c|c|c|c|c|c|c|c|c|c|c|c|c|c|c|c|c|}
\hline$P$ & 31 & 142 & 93 & 147 & 152 & 240 & 108 & 63 & 103 & 68 & 61 & 275 & 140 & 1660 & 212 & 72 & 113 & 158 & 125 \\
\hline $\mathrm{Zr}$ & 42.2 & 43.4 & 42.3 & 41.2 & 41.3 & 44.2 & 44.3 & 27.0 & 33.7 & 27.5 & 25.1 & 24.9 & 24.2 & 27.9 & 29.5 & 31.4 & 30.1 & 26.9 & 26.6 \\
\hline Y & 8.5 & 11.5 & 10.0 & 10.3 & 10.7 & 16.7 & 12.5 & 9.8 & 10.8 & 8.8 & 7.2 & 18.3 & 9.1 & 20.4 & 21.6 & 9.2 & 12.1 & 11.5 & 10.0 \\
\hline $\mathrm{Ga}$ & 14.2 & 11.5 & 15 & 11.6 & 14.9 & 18.2 & 18.1 & 14.3 & 18.1 & 15.6 & 13.7 & 14.6 & 12.3 & 15 & 15.1 & 15.9 & 16.8 & 14.4 & 14.3 \\
\hline $\mathrm{Rb}$ & 12.9 & 5.2 & 10.6 & 7.4 & 4.9 & 12.4 & 10.8 & 18.2 & 22.1 & 9.9 & 6.4 & 10.9 & 3.2 & 15.3 & 18.4 & 6.0 & 16.1 & 4.4 & 4.5 \\
\hline Sr & 129 & 90 & 125 & 76 & 123 & 156 & 160 & 147 & 162 & 172 & 157 & 135 & 122 & 163 & 158 & 163 & 222 & 141 & 141 \\
\hline $\mathrm{Ba}$ & 4 & 0 & 0 & 15 & 4 & 10 & 2 & 13 & 8 & 11 & 4 & 6 & 6 & 19 & 15 & 7 & 7 & 1 & 2 \\
\hline V & 176 & 255 & 191 & 201 & 210 & 209 & 213 & 195 & 209 & 203 & 204 & 221 & 221 & 221 & 234 & 225 & 233 & 216 & 214 \\
\hline $\mathrm{Nb}$ & 0.5 & 0.3 & 0.3 & 0.6 & 0.4 & 0.4 & 0.2 & 0.4 & 0.3 & 0.0 & 0.3 & 0.0 & 0.4 & 0.4 & 0.1 & 0.8 & 0.2 & 0.0 & 0.1 \\
\hline $\mathrm{Ni}$ & 55 & 52 & 51 & 45 & 56 & 40 & 44 & 103 & 59 & 111 & 127 & 150 & 146 & 110 & 112 & 89 & 78 & 170 & 168 \\
\hline $\mathrm{Cr}$ & 275 & 282 & 316 & 236 & 296 & 126 & 125 & 566 & 172 & 347 & 485 & 748 & 669 & 399 & 438 & 204 & 186 & 561 & 541 \\
\hline $\mathrm{Zn}$ & 77 & 64 & 67 & 56 & 68 & 74 & 79 & 68 & 72 & 77 & 68 & 74 & 65 & 62 & 68 & 77 & 70 & 68 & 65 \\
\hline $\mathrm{Cu}$ & 18 & 353 & 17 & 67 & 66 & 26 & 24 & 16 & 22 & 16 & 23 & 14 & 14 & 16 & 16 & 19 & 48 & 16 & 18 \\
\hline $\mathrm{Nd}$ & & & & & & 4.64 & & & & & & & & 5.92 & & & & & \\
\hline $\mathrm{Sm}$ & & & & & & 1.48 & & & & & & & & 1.73 & & & & & \\
\hline $100 \mathrm{Mg} / \mathrm{Mg}+\mathrm{Fe}(\mathrm{t})$ & 69.5 & 74.0 & 68.2 & 72.4 & 70.9 & 59.2 & 61.0 & 69.1 & 61.1 & 71.9 & 75.8 & 76.0 & 77.7 & 69.8 & 69.5 & 69.1 & 64.9 & 74.5 & 74.6 \\
\hline $\mathrm{Al} 2 \mathrm{O} 3 / \mathrm{TiO} 2$ & 46.6 & 44.8 & 46.4 & 45.1 & 44.7 & 49.9 & 50.2 & 48.6 & 51.3 & 49.0 & 47.8 & 48.3 & 47.8 & 46.2 & 50.2 & 51.5 & 51.4 & 47.1 & 47.0 \\
\hline $\mathrm{CaO} / \mathrm{TiO}_{2}$ & 31.5 & 28.8 & 31.3 & 27.4 & 31.2 & 27.8 & 27.8 & 33.0 & 29.6 & 32.1 & 34.6 & 40.8 & 40.2 & 34.1 & 36.1 & 30.5 & 28.4 & 37.9 & 36.7 \\
\hline $\mathrm{CaO} / \mathrm{Al} 2 \mathrm{O} 3$ & 0.68 & 0.64 & 0.67 & 0.61 & 0.70 & 0.56 & 0.55 & 0.68 & 0.58 & 0.65 & 0.72 & 0.85 & 0.84 & 0.74 & 0.72 & 0.59 & 0.55 & 0.80 & 0.78 \\
\hline $\mathrm{Ti} / \mathrm{Zr}$ & 48 & 47 & 47 & 48 & 46 & 47 & 50 & 58 & 57 & 63 & 62 & 60 & 68 & 62 & 62 & 63 & 64 & 68 & 66 \\
\hline $\mathrm{Zr} / \mathrm{Y}$ & 5.0 & 3.8 & 4.2 & 4.0 & 3.9 & 2.6 & 3.5 & 2.8 & 3.1 & 3.1 & 3.5 & 1.4 & 2.7 & 1.4 & 1.4 & 3.4 & 2.5 & 2.3 & 2.7 \\
\hline
\end{tabular}


Core, section

Depth (MBSF)

Sample no.

Unit

Lithology

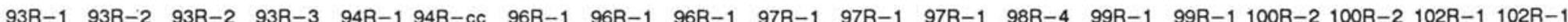
$\begin{array}{llllllllllllllllll}96-100 & 22-25 & 27-29 & 60 & 100-105118-126 & 77-81 & 81-83 & 93-98 & 80-85 & 98-102 & 124-127 & 63-67 & 54-55 & 140-144 & 16-19 & 28-30 & 36-40 & 128-132\end{array}$ $\begin{array}{llllllllllllllllllllllll}1472.86 & 1475.28 & 1475.44 & 1476.47 & 1483.21 & 1488.29 & 1504.07 & 1504.28 & 1505.16 & 1515.78 & 1517.31 & 1517.50 & 1526.61 & 1530.41 & 1534.84 & 1545.10 & 1545.20 & 1558.33 & 1562.51\end{array}$

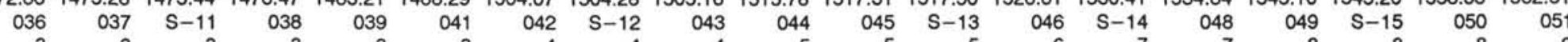
Breccia Breccia Breccia Breccia Breccia Breccia Pillow Pillow Pillow Massive Massive Massive Breccia Cpx-pill Cpx-pill Pillow Pillow Breccia Breccia

Major elements (wt\%)

\begin{tabular}{|c|c|c|c|c|c|c|c|c|c|c|c|c|c|c|c|c|c|c|c|}
\hline $\mathrm{SiO} 2$ & 52.73 & 53.51 & 53.41 & 54.37 & 53.71 & 55.15 & 53.14 & 53.48 & 54.36 & 52.64 & 53.17 & 53.19 & 55.03 & 53.02 & 53.66 & 53.96 & 53.50 & 54.05 & 55.26 \\
\hline $\mathrm{TiO} 2$ & 0.28 & 0.37 & 0.37 & 0.32 & 0.37 & 0.36 & 0.29 & 0.29 & 0.36 & 0.28 & 0.29 & 0.28 & 0.32 & 0.27 & 0.26 & 0.33 & 0.35 & 0.36 & 0.34 \\
\hline $\mathrm{Al} 2 \mathrm{O} 3$ & 12.76 & 17.90 & 17.71 & 16.07 & 18.17 & 17.70 & 14.15 & 14.46 & 17.97 & 13.97 & 14.88 & 13.83 & 16.91 & 13.50 & 13.34 & 17.19 & 18.13 & 17.37 & 16.37 \\
\hline $\mathrm{Fe} 2 \mathrm{O} 3$ * & 9.07 & 8.48 & 8.42 & 8.50 & 8.65 & 8.49 & 8.82 & 8.98 & 8.81 & 9.13 & 9.01 & 8.98 & 8.60 & 9.70 & 9.16 & 9.47 & 9.06 & 9.42 & 8.71 \\
\hline $\mathrm{MnO}$ & 0.19 & 0.15 & 0.15 & 0.16 & 0.14 & 0.16 & 0.18 & 0.16 & 0.13 & 0.20 & 0.19 & 0.20 & 0.15 & 0.16 & 0.17 & 0.13 & 0.13 & 0.11 & 0.15 \\
\hline $\mathrm{MgO}$ & 12.50 & 7.06 & 6.94 & 8.29 & 6.42 & 6.49 & 10.89 & 10.73 & 5.76 & 11.77 & 10.61 & 11.58 & 6.97 & 9.56 & 10.56 & 5.88 & 5.93 & 5.13 & 7.05 \\
\hline $\mathrm{CaO}$ & 10.04 & 10.37 & 10.29 & 9.54 & 9.96 & 9.35 & 10.42 & 10.57 & 9.68 & 9.97 & 10.01 & 9.92 & 8.19 & 10.82 & 9.99 & 9.52 & 10.20 & 9.20 & 8.80 \\
\hline $\mathrm{Na} 2 \mathrm{O}$ & 1.54 & 2.29 & 2.19 & 2.14 & 2.19 & 2.30 & 1.83 & 1.90 & 2.53 & 1.82 & 1.97 & 1.76 & 2.35 & 1.76 & 1.83 & 2.43 & 2.46 & 2.37 & 2.40 \\
\hline K2O & 0.23 & 0.37 & 0.47 & 0.60 & 0.47 & 0.65 & 0.33 & 0.36 & 0.89 & 0.22 & 0.26 & 0.21 & 0.77 & 0.90 & 0.67 & 0.91 & 0.72 & 1.51 & 0.73 \\
\hline P2O5 & 0.02 & 0.03 & 0.02 & 0.01 & 0.03 & 0.03 & 0.03 & 0.01 & 0.02 & 0.03 & 0.03 & 0.02 & 0.02 & 0.04 & 0.01 & 0.01 & 0.02 & 0.04 & 0.04 \\
\hline (LOI) & 1.68 & 2.55 & 1.93 & 3.23 & 1.47 & 3.74 & 2.76 & 1.68 & 1.55 & 1.27 & 1.65 & 1.55 & 4.07 & 1.62 & 1.82 & 1.83 & 0.81 & 1.71 & 2.42 \\
\hline Total & 99.36 & 100.53 & 99.97 & $\infty 0.00$ & 100.11 & 100.68 & 100.08 & 100.94 & 100.51 & 100.03 & 100.42 & 99.97 & 99.31 & 99.73 & 99.65 & 99.83 & 100.50 & 99.56 & 99.85 \\
\hline
\end{tabular}

Trace elements (ppm)

\begin{tabular}{|c|c|c|c|c|c|c|c|c|c|c|c|c|c|c|c|c|c|c|c|}
\hline $\mathrm{P}$ & 128 & 143 & 131 & 70 & 112 & 115 & 134 & 101 & 97 & 155 & 109 & 111 & 49 & 179 & 77 & 73 & 75 & 139 & 153 \\
\hline $\mathrm{Zr}$ & 26.3 & 36.3 & 34.6 & 31.4 & 35.8 & 34.6 & 28.8 & 29.1 & 35.2 & 27.8 & 29.5 & 27.6 & 32.4 & 26.6 & 26.1 & 33.3 & 34.4 & 37.5 & 35.9 \\
\hline Y & 9.7 & 11.9 & 9.8 & 10.3 & 12.2 & 11.1 & 11.3 & 11.1 & 11.6 & 13.0 & 10.0 & 8.8 & 8.5 & 11.5 & 9.3 & 10.1 & 10.6 & 13.6 & 12.5 \\
\hline Ga & 13.8 & 16.4 & & 15 & 17.5 & 16.3 & 14.9 & 15.1 & 18.8 & 14.4 & 14.9 & 13.5 & 15.9 & 15.6 & 14.5 & 17.3 & 17.4 & 18.3 & 15.9 \\
\hline $\mathrm{Rb}$ & 4.4 & 4.8 & 3.9 & 2.8 & 6.8 & 6.6 & 5.8 & 7.2 & 13.4 & 4.4 & 3.2 & 3.5 & 9.6 & 17.2 & 12.8 & 16.4 & 12.0 & 27.1 & 7.2 \\
\hline $\mathrm{Sr}$ & 141 & 190 & 184 & 154 & 189 & 164 & 149 & 152 & 193 & 149 & 151 & 140 & 176 & 140 & 132 & 187 & 197 & 184 & 152 \\
\hline $\mathrm{Ba}$ & 2 & 0 & 13 & 8 & 5 & 5 & 0 & 5 & 9 & 1 & 0 & 3 & 23 & 11 & 12 & 0 & 4 & 0 & 1 \\
\hline V & 216 & 259 & 260 & 214 & 252 & 231 & 231 & 230 & 222 & 221 & 237 & 211 & 215 & 256 & 229 & 233 & 247 & 244 & 254 \\
\hline $\mathrm{Nb}$ & 0.5 & 0.4 & 0.5 & 0.2 & 0.4 & 0.1 & 0.1 & 0.6 & 0.0 & 0.1 & 0.5 & 0.4 & 0.1 & 0.6 & 0.5 & 0.5 & 0.5 & 0.4 & $=0.4$ \\
\hline $\mathrm{Ni}$ & 161 & 56 & 55 & 78 & 46 & 42 & 138 & 140 & 45 & 127 & 107 & 123 & 39 & 122 & 137 & 59 & 48 & 51 & 46 \\
\hline $\mathrm{Cr}$ & 491 & 174 & 144 & 260 & 74 & 73 & 569 & 544 & 70 & 432 & 390 & 488 & 39 & 596 & 491 & 62 & 87 & 126 & 162 \\
\hline $\mathrm{Zn}$ & 66 & 70 & 88 & 71 & 72 & 67 & 83 & 79 & 76 & 67 & 66 & 64 & 69 & 75 & 79 & 85 & 87 & 75 & 65 \\
\hline $\mathrm{Cu}$ & 18 & 72 & 76 & 19 & 25 & 45 & 18 & 19 & 19 & 22 & 16 & 24 & 129 & 15 & 34 & 21 & 19 & 18 & 164 \\
\hline $\mathrm{Nd}$ & & & & & & & & 3.63 & & & & 2.46 & & 3.69 & & & & & \\
\hline $\mathrm{Sm}$ & & & & & & & & 1.20 & & & & 0.84 & & 1.21 & & & & & \\
\hline $100 \mathrm{Mg} / \mathrm{Mg}+\mathrm{Fe}(\mathrm{t})$ & 75.2 & 64.7 & 64.5 & 68.2 & 62.0 & 62.7 & 73.1 & 72.5 & 59.0 & 73.9 & 72.2 & 73.9 & 64.1 & 68.4 & 71.7 & 57.7 & 59.0 & 54.5 & 64.0 \\
\hline $\mathrm{Al} 2 \mathrm{O} 3 / \mathrm{TTO} 2$ & 45.6 & 48.4 & 47.9 & 50.2 & 49.1 & 49.2 & 48.8 & 49.9 & 49.9 & 49.9 & 51.3 & 49.4 & 52.8 & 50.0 & 51.3 & 52.1 & 51.8 & 48.3 & 48.1 \\
\hline $\mathrm{CaO} / \mathrm{TiO}$ & 35.9 & 28.0 & 27.8 & 29.8 & 26.9 & 26.0 & 35.9 & 36.4 & 26.9 & 35.6 & 34.5 & 35.4 & 25.6 & 40.1 & 38.4 & 28.8 & 29.1 & 25.6 & 25.9 \\
\hline $\mathrm{CaO} / \mathrm{Al} 2 \mathrm{O} 3$ & 0.79 & 0.58 & 0.58 & 0.59 & 0.55 & 0.53 & 0.74 & 0.73 & 0.54 & 0.71 & 0.67 & 0.72 & 0.48 & 0.80 & 0.75 & 0.55 & 0.56 & 0.53 & 0.54 \\
\hline $\mathrm{Ti} / \mathrm{Zr}$ & 69 & 64 & 64 & 63 & 62 & 60 & 64 & 60 & 61 & 62 & 63 & 61 & 59 & 63 & 65 & 61 & 60 & 59 & 58 \\
\hline $\mathrm{Zr} / \mathrm{Y}$ & 2.7 & 3.1 & 2.5 & 3.0 & 2.9 & 3.1 & 2.5 & 2.6 & 3.0 & 2.1 & 3.0 & 3.1 & 3.8 & 2.3 & 2.8 & 3.3 & 3.2 & 2.8 & 2.9 \\
\hline
\end{tabular}


Core, section
Interval

Depth (MBSF)

Sample no.

Unit

Lithology
103R-1 104R-1 104R-1 104R-2 104R-2 104R-3 105R-1 105R-1 105R-3 106R-2 107R-1 107R-1 107R-3 108R-1 108R-2 109R-2 109R-3 109R-3 110R-1

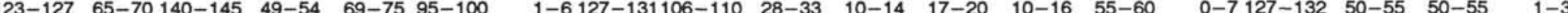

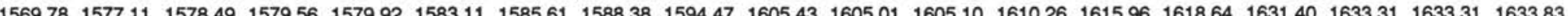

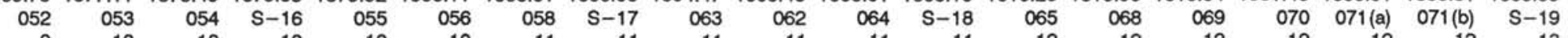
Breccia Massive Massive Massive Massive Massive Pillow Pillow Pillow Pillow Pillow Pillow Breccia Breccia Breccia Breccia Breccia Breccia massive

Major elements (wt\%)

\begin{tabular}{|c|c|c|c|c|c|c|c|c|c|c|c|c|c|c|c|c|c|c|c|}
\hline $\mathrm{SiO} 2$ & 53.95 & 52.80 & 52.88 & 53.18 & 53.37 & 53.69 & 55.20 & 56.04 & 54.41 & 54.01 & 57.26 & 53.51 & 53.34 & 53.28 & 52.53 & 53.07 & 56.13 & 54.28 & 54.56 \\
\hline TIO2 & 0.37 & 0.30 & 0.31 & 0.32 & 0.31 & 0.29 & 0.30 & 0.28 & 0.29 & 0.29 & 0.29 & 0.33 & 0.33 & 0.27 & 0.30 & 0.29 & 0.27 & 0.26 & 0.25 \\
\hline $\mathrm{Al} 2 \mathrm{O} 3$ & 16.83 & 13.68 & 14.28 & 14.55 & 14.45 & 13.54 & 13.74 & 13.03 & 13.46 & 14.57 & 15.26 & 16.78 & 14.73 & 14.64 & 14.70 & 14.40 & 14.04 & 13.50 & 13.02 \\
\hline $\mathrm{Fe} 2 \mathrm{O} 3$ * & 9.63 & 9.36 & 9.63 & 9.17 & 8.90 & 9.48 & 8.96 & 9.09 & 9.35 & 9.51 & 7.68 & 9.35 & 8.97 & 8.49 & 8.59 & 8.77 & 8.01 & 8.66 & 9.14 \\
\hline $\mathrm{MnO}$ & 0.14 & 0.16 & 0.17 & 0.16 & 0.14 & 0.17 & 0.17 & 0.17 & 0.17 & 0.17 & 0.13 & 0.14 & 0.17 & 0.16 & 0.16 & 0.17 & 0.14 & 0.17 & 0.15 \\
\hline $\mathrm{MgO}$ & 7.27 & 9.76 & 8.99 & 8.23 & 7.41 & 9.37 & 8.37 & 8.66 & 8.61 & 8.28 & 4.64 & 7.34 & 9.88 & 11.26 & 10.67 & 11.30 & 9.83 & 11.79 & 10.15 \\
\hline $\mathrm{CaO}$ & 8.90 & 11.66 & 11.22 & 11.62 & 11.57 & 11.52 & 10.54 & 10.11 & 10.75 & 10.63 & 9.61 & 10.20 & 10.39 & 9.42 & 10.42 & 9.95 & 8.41 & 9.25 & 9.83 \\
\hline $\mathrm{Na} 2 \mathrm{O}$ & 2.31 & 1.78 & 2.03 & 2.17 & 2.27 & 1.98 & 2.24 & 2.04 & 1.77 & 2.15 & 2.74 & 2.26 & 1.81 & 1.89 & 1.87 & 1.86 & 2.05 & 1.73 & 2.29 \\
\hline $\mathrm{K} 2 \mathrm{O}$ & 0.55 & 0.28 & 0.29 & 0.51 & 0.91 & 0.20 & 0.46 & 0.46 & 0.64 & 0.24 & 1.39 & 0.50 & 0.32 & 0.26 & 0.26 & 0.22 & 0.58 & 0.34 & 0.69 \\
\hline P2O5 & 0.02 & 0.02 & 0.03 & 0.06 & 0.13 & 0.03 & 0.03 & 0.03 & 0.03 & 0.03 & 0.51 & 0.02 & 0.03 & 0.02 & 0.04 & 0.02 & 0.03 & 0.02 & 0.04 \\
\hline (LOI) & 1.24 & 1.30 & 1.42 & 0.83 & 1.69 & 2.14 & 2.60 & 2.27 & 2.31 & 1.32 & 0.48 & 1.42 & 1.31 & 1.99 & 1.65 & 1.33 & 1.96 & 1.59 & 0.77 \\
\hline Total & 99.97 & 99.80 & 99.83 & 99.97 & 99.46 & 100.27 & 100.01 & 99.91 & 99.48 & 99.88 & 99.51 & 100.43 & 99.97 & 99.69 & 99.54 & 100.05 & 99.49 & 100.00 & 100.12 \\
\hline
\end{tabular}

Trace elements (ppm)

$\mathrm{P}$
$\mathrm{Zr}$
$\mathrm{Y}$
$\mathrm{Ga}$
$\mathrm{Rb}$
$\mathrm{Sr}$
$\mathrm{Ba}$
$\mathrm{V}$
$\mathrm{Nb}$
$\mathrm{Ni}$
$\mathrm{Cr}$
$\mathrm{Zn}$
$\mathrm{Cu}$
$\mathrm{Nd}$
$\mathrm{Sm}$

$100 \mathrm{Mg} / \mathrm{Mg}+\mathrm{Fe}(\mathrm{t})$ $\mathrm{Al} 2 \mathrm{O} 3 \mathrm{TiO} 2$

$\mathrm{CaO} / \mathrm{TiO} 2$

$\mathrm{CaO} / \mathrm{Al} 2 \mathrm{O} 3$

$\mathrm{Ti} / \mathrm{Zr}$

$\mathrm{Zr} N$

$\begin{array}{rr}163 & 292 \\ 32.1 & 33.5 \\ 11.7 & 15.4 \\ 15.2 & 16.3 \\ 4.7 & 9.6 \\ 174 & 178 \\ 3 & 7 \\ 259 & 259 \\ 0.3 & 0.5 \\ 45 & 49 \\ 240 & 270 \\ 81 & 76 \\ 29 & 18 \\ & 5.61 \\ & 1.78\end{array}$

754
31.2
21.7
16.8
16.9
201
14
264
0.1
49
278
77
57

$\begin{array}{rr}145 & 150 \\ 30.1 & 33.0 \\ 9.8 & 10.6 \\ 14.3 & 15 \\ 2.5 & 5 \\ 234 & 145 \\ 12 & \\ 244 & 239 \\ 0.5 & 0.4 \\ 44 & \\ 235 & 189 \\ 66 & \\ 53 & 139\end{array}$

$\begin{array}{rr}62.4 & 69.7 \\ 45.5 & 45.6 \\ 24.1 & 38.9 \\ 0.53 & 0.8 \\ 59 & 6\end{array}$
$67.3 \quad 66.4$

$\begin{array}{ll}46.1 & 45.5 \\ 36.2 & 36.3\end{array}$

$36.2 \quad 36.3$
64
46
37
0.80
1 $\begin{array}{rr}64.7 & 68.5 \\ 46.6 & 46.7 \\ 37.3 & 39.7 \\ 0.80 & 0.85 \\ 68 & \\ 1.4 & \end{array}$

33.0
10.6
5.2
5.0
145
8
239
0.4
34
189
66
139

$\begin{array}{rrrr}125 & 142 & 208 & 2350 \\ 31.2 & 31.5 & 34.7 & 34.0 \\ 10.4 & 10.0 & 12.1 & 50.9 \\ 12.7 & 15.7 & 15.5 & 15.9 \\ 5.8 & 10.0 & 4.3 & 22.5 \\ 129 & 157 & 180 & 197 \\ 6 & 10 & 10 & 38 \\ 231 & 225 & 245 & 221 \\ 0.5 & 0.0 & 0.6 & 0.0 \\ 38 & 45 & 36 & 27 \\ 221 & 280 & 159 & 139 \\ 64 & 72 & 71 & 58 \\ 72 & 91 & 23 & 98\end{array}$

$\begin{array}{rr}77 & 118 \\ 34.7 & 33.3 \\ 9.9 & 11.2 \\ 17.2 & 14.6 \\ 8.7 & 6.2 \\ 183 & 150 \\ 7 & 0 \\ 237 & 235 \\ 0.4 & 0.6 \\ 37 & 106 \\ 162 & 320 \\ 86 & 77 \\ 169 & 16\end{array}$

$\begin{array}{rr}96 & 181 \\ 30.7 & 28.1 \\ 9.9 & 11 \\ 14.8 & 15 \\ 4.2 & 5.0 \\ 153 & 147 \\ 4 & \\ 205 & 233 \\ 0.0 & 0.0 \\ 134 & 124 \\ 400 & 351 \\ 65 & \\ 55 & \end{array}$

\begin{tabular}{rr}
181 & 114 \\
28.1 & 28. \\
11.6 & 10.0 \\
15.8 & 16.4 \\
5.0 & 5.0 \\
147 & 14 \\
2 & \\
233 & 21 \\
0.0 & 0.2 \\
124 & 123 \\
351 & 345 \\
70 & \\
20 & \\
\hline
\end{tabular}

$\begin{array}{rrrr}114 & 101 & 74 & 201 \\ 28.1 & 31.0 & 29.7 & 25.1 \\ 10.0 & 9.8 & 10.1 & 13.0 \\ 16.4 & 13.1 & 13 & 13.5 \\ 5.0 & 9.7 & 4.8 & 11.5 \\ 145 & 153 & 129 & 147 \\ 1 & 28 & 0 & 20 \\ 218 & 189 & 200 & 249 \\ 0.2 & 0.1 & 0.0 & 0.5 \\ 123 & 123 & 132 & 124 \\ 345 & 449 & 490 & 647 \\ 68 & 59 & 64 & 65 \\ 43 & 68 & 120 & 102 \\ & & & 3.46 \\ & & & 1.06\end{array}$

$68.5 \quad 67.3$ 0.97

1.06

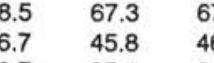

$\begin{array}{lllllllllll}67.0 & 65.7 & 57.1 & 63.3 & 70.8 & 74.5 & 73.2 & 73.9 & 73.0 & 75.0 & 71.0\end{array}$

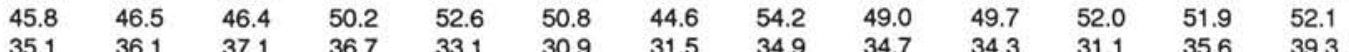

$\begin{array}{lllllllllllll}0.77 & 0.78 & 0.80 & 0.73 & 0.63 & 0.61 & 0.71 & 0.64 & 0.71 & 0.69 & 0.60 & 0.69 & 0.75\end{array}$

$\begin{array}{rrr}60 & 57 & 62 \\ 3.1 & 3.0 & 3.2\end{array}$


Core, section $\quad 110 R-1 \quad 110 R-2 \quad 110 R-2 \quad 110 R-3 \quad 110 R-4 \quad 110 R-5 \quad 111 R-1 \quad 111 R-2 \quad 111 R-2 \quad 112 R-1 \quad 112 R-1 \quad 112 R-1 \quad 112 R-2 \quad 112 R-2 \quad 113 R-2 \quad 113 R-2 \quad 113 R-3 \quad 113 R-3 \quad 113 R-4$ 110R-1 110R-2 110R-2 110R-3 110R-4 110R-5 111R-1 $111 R-2$ - $111 R-2$ 112R-1 112R-1 112R-1 112R-2 112R-2 113R-2 113R-2 113R-3 113R-3 $113 R-4$

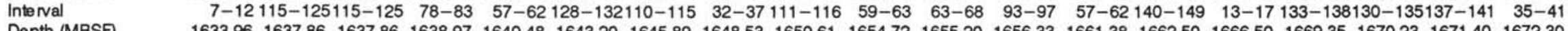

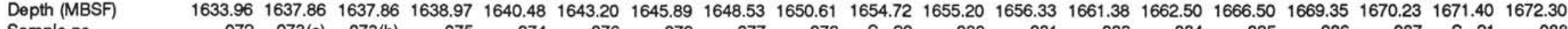
Sample no.

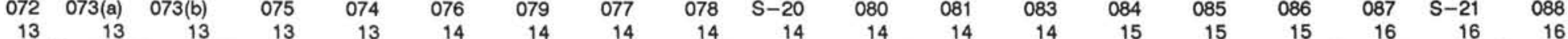
Unit

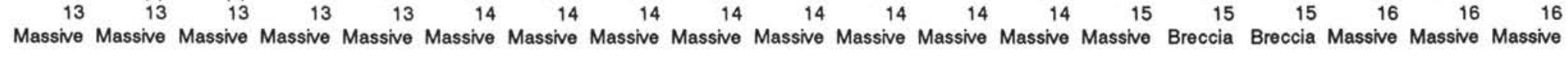

Lithology

Major elements (wt\%)

\begin{tabular}{|c|c|c|c|c|c|c|c|c|c|c|c|c|c|c|c|c|c|c|c|}
\hline $\mathrm{SiO} 2$ & 53.99 & 55.44 & 55.12 & 54.41 & 53.72 & 56.71 & 54.82 & 52.93 & 54.30 & 55.51 & 56.77 & 55.53 & 53.75 & 57.92 & 52.81 & 52.97 & 57.83 & 59.29 & 58.84 \\
\hline $\mathrm{TiO} 2$ & 0.25 & 0.26 & 0.25 & 0.26 & 0.24 & 0.31 & 0.26 & 0.24 & 0.24 & 0.30 & 0.29 & 0.28 & 0.25 & 0.32 & 0.30 & 0.31 & 0.32 & 0.31 & 0.30 \\
\hline $\mathrm{Al} 2 \mathrm{O} 3$ & 12.33 & 12.93 & 12.29 & 12.66 & 12.24 & 15.92 & 13.17 & 11.91 & 11.37 & 15.08 & 15.04 & 13.96 & 12.58 & 16.32 & 16.36 & 16.86 & 16.74 & 16.61 & 16.83 \\
\hline $\mathrm{Fe} 2 \mathrm{O} 3$ * & 8.80 & 8.94 & 9.79 & 9.18 & 8.94 & 8.59 & 9.12 & 9.49 & 8.59 & 8.00 & 7.99 & 8.61 & 8.84 & 7.62 & 9.23 & 8.57 & 8.07 & 7.51 & 7.51 \\
\hline $\mathrm{MnO}$ & 0.15 & 0.14 & 0.15 & 0.15 & 0.16 & 0.11 & 0.13 & 0.14 & 0.16 & 0.12 & 0.12 & 0.12 & 0.16 & 0.10 & 0.15 & 0.13 & 0.10 & 0.09 & 0.09 \\
\hline $\mathrm{MgO}$ & 11.08 & 9.14 & 9.62 & 9.64 & 10.49 & 4.24 & 9.78 & 13.31 & 13.61 & 7.06 & 6.35 & 7.23 & 12.51 & 3.55 & 8.84 & 8.07 & 3.51 & 3.24 & 3.28 \\
\hline $\mathrm{CaO}$ & 10.09 & 9.63 & 9.74 & 9.99 & 11.04 & 8.86 & 9.01 & 9.22 & 9.07 & 9.28 & 8.99 & 9.27 & 9.03 & 8.36 & 9.37 & 9.61 & 8.37 & 8.16 & 8.22 \\
\hline $\mathrm{Na} 2 \mathrm{O}$ & 2.15 & 2.42 & 2.36 & 2.32 & 2.22 & 3.11 & 2.42 & 1.76 & 1.91 & 2.71 & 2.80 & 2.52 & 2.04 & 3.09 & 2.15 & 2.47 & 3.10 & 3.03 & 3.14 \\
\hline $\mathrm{K} 2 \mathrm{O}$ & 0.60 & 0.77 & 0.88 & 0.75 & 0.77 & 1.48 & 0.96 & 0.60 & 0.33 & 1.19 & 1.37 & 1.51 & 0.35 & 1.69 & 0.31 & 0.50 & 1.85 & 1.69 & 1.72 \\
\hline P2O5 & 0.03 & 0.04 & 0.04 & 0.04 & 0.04 & 0.09 & 0.05 & 0.05 & 0.03 & 0.69 & 0.52 & 0.66 & 0.11 & 0.35 & 0.02 & 0.01 & 0.06 & 0.06 & 0.06 \\
\hline (LOI) & 1.34 & 1.19 & 1.49 & 1.87 & 1.81 & 1.12 & 1.68 & 2.35 & 1.60 & 0.90 & 0.86 & 1.48 & 1.46 & 1.41 & 1.91 & 1.68 & 0.83 & 0.64 & 1.04 \\
\hline Total & 99.47 & 99.71 & 100.24 & 99.40 & 99.86 & 99.42 & 99.72 & 99.65 & 99.61 & 99.94 & 100.24 & 99.69 & 99.62 & 99.32 & 99.54 & 99.50 & 99.95 & 99.99 & 99.9 \\
\hline
\end{tabular}

Trace elements (ppm)

\begin{tabular}{|c|c|c|c|c|c|c|c|c|c|c|c|c|c|c|c|c|c|c|c|}
\hline$P$ & 195 & 205 & 194 & 191 & 185 & 434 & 248 & 292 & 160 & 3589 & 2815 & 3422 & 637 & 1829 & 94 & 80 & 269 & 279 & 254 \\
\hline $\mathrm{Zr}$ & 24.1 & 25.9 & 24.4 & 24.5 & 23.2 & 30.8 & 25.4 & 23.0 & 23.5 & 29.7 & 30.5 & 27.9 & 25.7 & 33.4 & 33.7 & 33.9 & 35.5 & 34.4 & 33.4 \\
\hline Y & 12.3 & 12.8 & 12.5 & 12.4 & 11.3 & 20.7 & 11.0 & 15.4 & 8.9 & 18.5 & 21.3 & 24.3 & 11.7 & 49.7 & 9.6 & 8.5 & 21.9 & 21.6 & 21.9 \\
\hline $\mathrm{Ga}$ & 13.7 & 14.5 & 13.7 & 13.8 & 13.9 & 16.4 & 14.6 & 13.1 & 12.2 & 14.4 & 14.9 & 14.2 & 13.1 & 16.3 & 16.3 & 16.8 & 19.1 & 18.5 & 17.1 \\
\hline $\mathrm{Rb}$ & 10.1 & 15.2 & 17.8 & 12.7 & 13.2 & 33.0 & 20.6 & 13.3 & 7.4 & 25.3 & 31.7 & 36.9 & 7.0 & 33.5 & 5.4 & 8.9 & 35.4 & 31.5 & 31.3 \\
\hline Sr & 142 & 151 & 136 & 143 & 139 & 191 & 152 & 131 & 130 & 187 & 183 & 172 & 143 & 197 & 184 & 182 & 197 & 196 & 195 \\
\hline $\mathrm{Ba}$ & 5 & 14 & 26 & 18 & 21 & 13 & 20 & 0 & 19 & 22 & 25 & 19 & 16 & 21 & 8 & 8 & 32 & 25 & 17 \\
\hline V & 219 & 221 & 215 & 214 & 248 & 221 & 202 & 188 & 209 & 221 & 216 & 196 & 215 & 219 & 247 & 249 & 237 & 227 & 220 \\
\hline $\mathrm{Nb}$ & 0.0 & 0.0 & 0.3 & 0.2 & 0.3 & 0.0 & 0.2 & 0.6 & 0.4 & 0.3 & 0.0 & 0.3 & 0.5 & 0.0 & 0.2 & 0.0 & 0.2 & 0.2 & 0.3 \\
\hline $\mathrm{Ni}$ & 139 & 90 & 114 & 100 & 112 & 40 & 126 & 146 & 158 & 83 & 71 & 89 & 143 & 36 & 81 & 87 & 28 & 23 & 21 \\
\hline $\mathrm{Cr}$ & 637 & 453 & 737 & 563 & 606 & 92 & 493 & 565 & 670 & 239 & 222 & 366 & 536 & 56 & 219 & 238 & 41 & 40 & 33 \\
\hline $\mathrm{Zn}$ & 64 & 59 & 64 & 57 & 57 & 61 & 65 & 68 & 65 & 58 & 53 & 55 & 70 & 51 & 74 & 75 & 58 & 51 & 51 \\
\hline $\mathrm{Cu}$ & 28 & 36 & 45 & 130 & 44 & 112 & 14 & 6 & 54 & 19 & 22 & 25 & 12 & 21 & 142 & 361 & 15 & 38 & 25 \\
\hline $\mathrm{Nd}$ & & & & & & & & & & 3.28 & & & & & & & & 7.36 & \\
\hline $\mathrm{Sm}$ & & & & & & & & & & 1.04 & & & & & & & & 2.32 & \\
\hline $100 \mathrm{Mg} / \mathrm{Mg}+\mathrm{Fe}(\mathrm{t})$ & 73.5 & 69.2 & 68.4 & 69.8 & 72.1 & 52.1 & 70.2 & 75.5 & 77.7 & 66.0 & 63.6 & 64.9 & 75.7 & 50.6 & 67.8 & 67.5 & 48.9 & 48.7 & 49.0 \\
\hline $\mathrm{Al} 2 \mathrm{O} / \mathrm{TTO} 2$ & 49.3 & 49.7 & 49.2 & 48.7 & 51.0 & 51.4 & 50.7 & 49.6 & 47.4 & 50.3 & 51.9 & 49.9 & 50.3 & 51.0 & 54.5 & 54.4 & 52.3 & 53.6 & 56.1 \\
\hline $\mathrm{CaO} / \mathrm{TIO} 2$ & 40.4 & 37.0 & 39.0 & 38.4 & 46.0 & 28.6 & 34.7 & 38.4 & 37.8 & 30.9 & 31.0 & 33.1 & 36.1 & 26.1 & 31.2 & 31.0 & 26.2 & 26.3 & 27.4 \\
\hline $\mathrm{CaO} / \mathrm{Al} 2 \mathrm{O} 3$ & 0.82 & 0.74 & 0.79 & 0.79 & 0.90 & 0.56 & 0.68 & 0.77 & 0.80 & 0.62 & 0.60 & 0.66 & 0.72 & 0.51 & 0.57 & 0.57 & 0.50 & 0.49 & 0.49 \\
\hline $\mathrm{Ti} / \mathrm{Zr}$ & 65 & 63 & 62 & 58 & 70 & 58 & 65 & 67 & 66 & 61 & 59 & 59 & 63 & 57 & 60 & 63 & 56 & 55 & 54 \\
\hline $\mathrm{Zr} / \mathrm{Y}$ & 2.0 & 2.0 & 2.0 & 2.0 & 2.1 & 1.5 & 2.3 & 1.5 & 2.6 & 1.6 & 1.4 & 1.1 & 2.2 & 0.7 & 3.5 & 4.0 & 1.6 & 1.6 & 1.5 \\
\hline
\end{tabular}


Table 3. Least squares mixing calculations, Hole 793B basement.

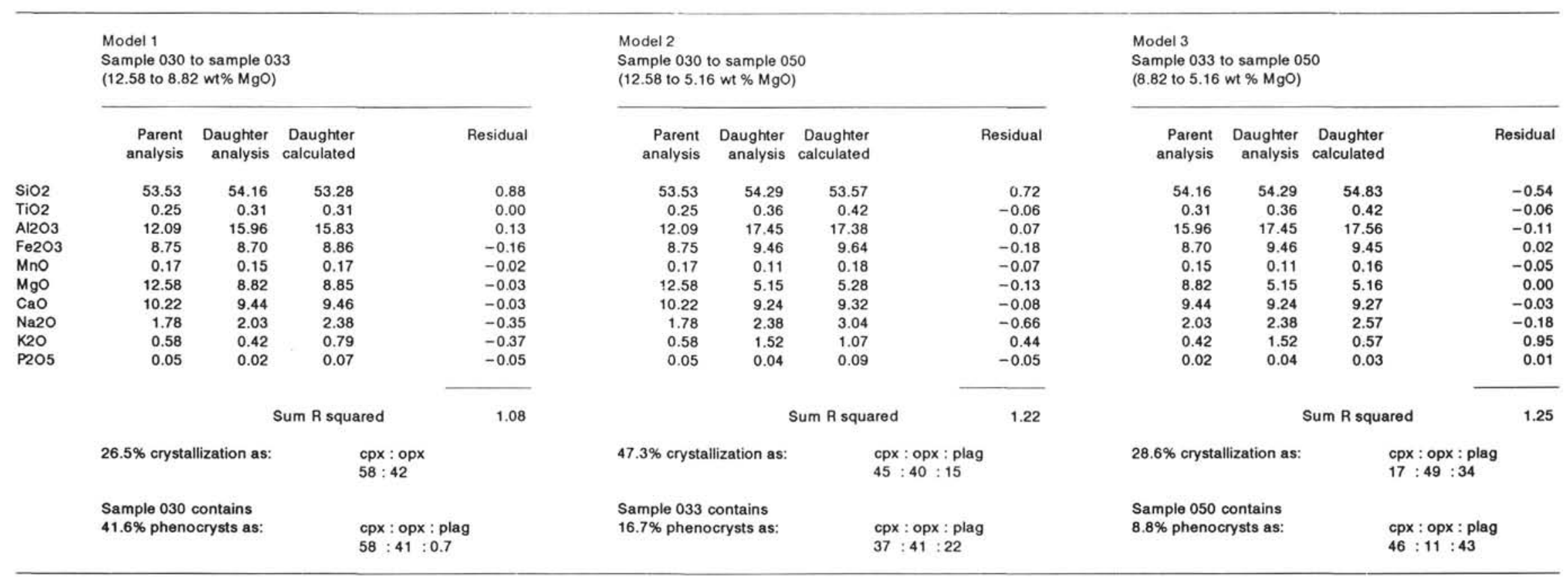

Notes: Modal proportions calculated on a vesicle-free basis. Mineral compositions taken as averages of phenocryst analyses from sample 033 (Lapierre et al., this volume). 

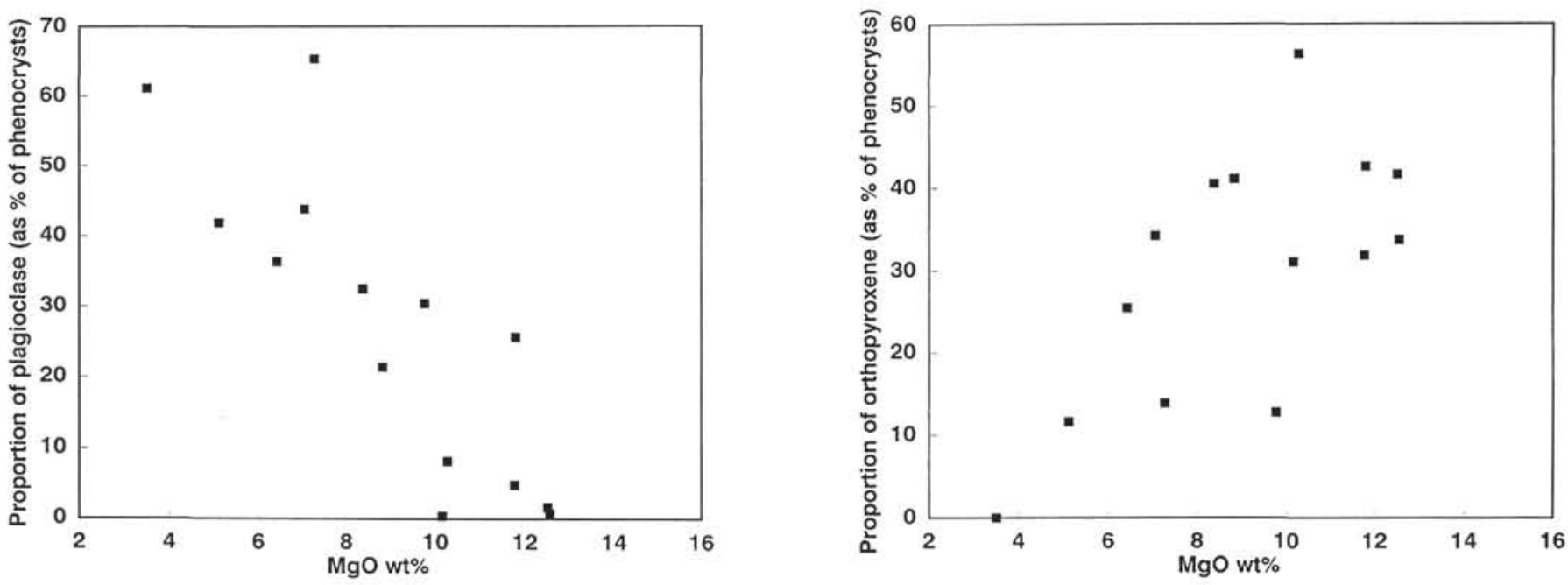

Figure 5. MgO contents of Hole 793B basement plotted vs. the proportion of plagioclase and orthopyroxene expressed as a percentage of total phenocrysts. Modal analyses are given in Table 1.

lavas of Group B (see also Fig. 4). Clinopyroxene analyses from Group A lavas reflect the whole-rock compositions in having appropriately lower Fe/Mg ratios and total Fe contents (see Lapierre et al., this volume). Both groups show positive correlations between $\mathrm{Zr}$ and $\mathrm{Ti}, \mathrm{Ga}, \mathrm{Sr}$, and $\mathrm{Al}_{2} \mathrm{O}_{3}$, with inter-element ratios remaining roughly constant. $\mathrm{Ni}$ and $\mathrm{Cr}$ correlate negatively with $\mathrm{Zr}$, reflecting the removal or addition of pyroxenes.

\section{Aspects of Trace Element Enrichment}

Relationships between $\mathrm{Zr}$ and certain other trace elements are not as easily explained. On Figure 6, P and $\mathrm{Y}$ show no correlation with $\mathrm{Zr}$ and $\mathrm{Sm}+\mathrm{Nd}$ a very weak one. Yttrium varies by a factor of 5 and $\mathrm{P}$ by a factor of 30 in samples ranging from 25 to $35 \mathrm{ppm} \mathrm{Zr}$. Similar, though less pronounced discrepancies, are noted in the combined Nd and Sm against $\mathrm{Zr}$ plot. Figure 7 demonstrates the anomalous behavior of certain trace elements. On this plot, concentrations of a low- $\mathrm{MgO}$ (anomalous) sample (S-21: 126-793B-113R-3, 137-141 cm) are normalized to a primitive high-MgO sample (S-13: 126-793B-98R-3, 124-127 cm). Also shown for comparison on Figure 7 are predicted trace element concentrations after removal of $30 \%$ and $80 \%$ of observed phenocrysts in $\mathrm{S}-13$. Of the trace elements, only $\mathrm{Sr}, \mathrm{Ti}, \mathrm{Zr}$, and $\mathrm{Ga}$ are close to predicted concentrations after $30 \%$ crystallization. Levels of P, Y, Nd, Sm, and the low-field-strength elements (LFSE) are all significantly higher, requiring in excess of $70 \%$ crystallization to generate the observed concentrations. REE patterns from two basement samples (Table 4 and Fig. 8) show nonparallel profiles, suggesting a process other than crystallization relates lavas within the sequence.

As elements such as $\mathrm{P}, \mathrm{Y}$, and the REE are normally considered to be immobile during low-temperature hydrothermal alteration (e.g., Saunders et al., 1980), the possibility of a primary magmatic origin for the trace element peculiarities must be examined. Because lower $\mathrm{MgO}$ samples tend to be the most affected by $\mathrm{P}$ and $\mathrm{Y}$ enrichment, it is possible that an assimilation/crystallization or magma mixing process may be responsible. To test this hypothesis, a single massive flow containing a range of phenocryst abundances and $\mathrm{MgO}$ contents was examined. Subunit 14a (Fig. 3) is a suitable flow with recognizable upper and lower contacts and good recovery. Six whole-rock samples were analyzed from this flow with a view to examining its internal chemical variations.

Geochemical profiles throughout the Subunit 14a flow are shown in Figure 9. Phenocrysts tend to be concentrated in the center of the unit, reflecting a flowage differentiation during extrusion. Variations within major element, $\mathrm{Zr}, \mathrm{Ga}, \mathrm{Cr}, \mathrm{Ni}, \mathrm{Sr}$, and $\mathrm{V}$ profiles can be explained by dilution of the erupted liquid by the observed phenocryst abundances. However, the profiles for $\mathrm{P}, \mathrm{Y}, \mathrm{K}_{2} \mathrm{O}$, and $\mathrm{Rb}$ do not fit a simple dilution model. $\mathrm{K}_{2} \mathrm{O}$ and $\mathrm{Rb}$ are enriched by factors of 3-4 between the low- and high-MgO samples, far greater than the enrichment factors of $1.3-1.5$ for $\mathrm{Sr}, \mathrm{Ti}, \mathrm{Zr}$, and $\mathrm{Ga}$. Phosphorus and yttrium are also over-enriched in the lower $\mathrm{MgO}$ samples, however, in contrast to $\mathrm{Rb}$ and $\mathrm{K}_{2} \mathrm{O}$, concentrations of these elements are greatest in the two samples from the base of the flow. The enrichment in P between the center and the base of the flow is by factors of 18-22. Similar $\mathrm{P}$ and $\mathrm{Y}$ anomalies were found throughout the section but are only recognized within massive and pillowed flows and were absent in samples taken from breccia units (see $\mathrm{P}-\mathrm{Zr}$ and $\mathrm{Y}-\mathrm{Zr}$ plots, Fig. 6).

In light of the known mobility of elements such as $\mathrm{K}$ and $\mathrm{Rb}$ during alteration, enrichment in the low-MgO sections of Subunit 14a can be explained by hydrothermal redistribution of these elements within the flow. On a broad scale, samples with high $\mathrm{K}_{2} \mathrm{O}$ also tend to have greater concentrations of $\mathrm{P}$ and $\mathrm{Y}$. However, the over-enrichment of $\mathrm{P}$ at the base of Subunit 14a suggests that another event or process may have disturbed the original igneous concentrations. Clearly, assimilation of host lithologies, either before or after eruption, is unlikely to produce such extreme enrichment within a single flow.

The most likely explanation for anomalous $\mathrm{P}, \mathrm{Y}$, and the rare-earth element (REE) concentrations relative to HFS elements is a low-temperature hydrothermal alteration process. Bienvenu et al. (1990) have described the systematic removal of REE, Y, and $\mathrm{P}$ and retention of $\mathrm{Zr}$ and Ti during alteration of mid-ocean ridge basalt (MORB) glasses to smectite. In the case of these oceanic lavas, the trivalent elements and $\mathrm{P}$ were removed from the system during fluid (seawater) interaction. In contrast, $\mathrm{Zr}$ and $\mathrm{Ti}$ remained in the glass/smectite system because of their high ionic potential and hydrolytic properties. Similar smectitization took place in Hole 793B lavas during hydrothermal circulation within the basement after eruption. However, the heterogeneous nature of the Hole 793B basement (namely, the intercalations of breccias and flows) has clearly affected fluid flow and element redistribution. Breccia units, which lack the anomalous P, Y, and REE values (Fig. 6), may act as permeable pathways for the efficient removal of fluids from the system without deposition of the solute. In contrast, massive and pillowed flows are less permeable and simply redistribute the mobilized elements internally. This has produced regions within flows where element leaching has occurred, and other areas (notably the base of Subunit 14a) where precipitation is dominant. The behavior of $\mathrm{Mg}, \mathrm{Fe}$, and $\mathrm{Al}$ with the sequence 

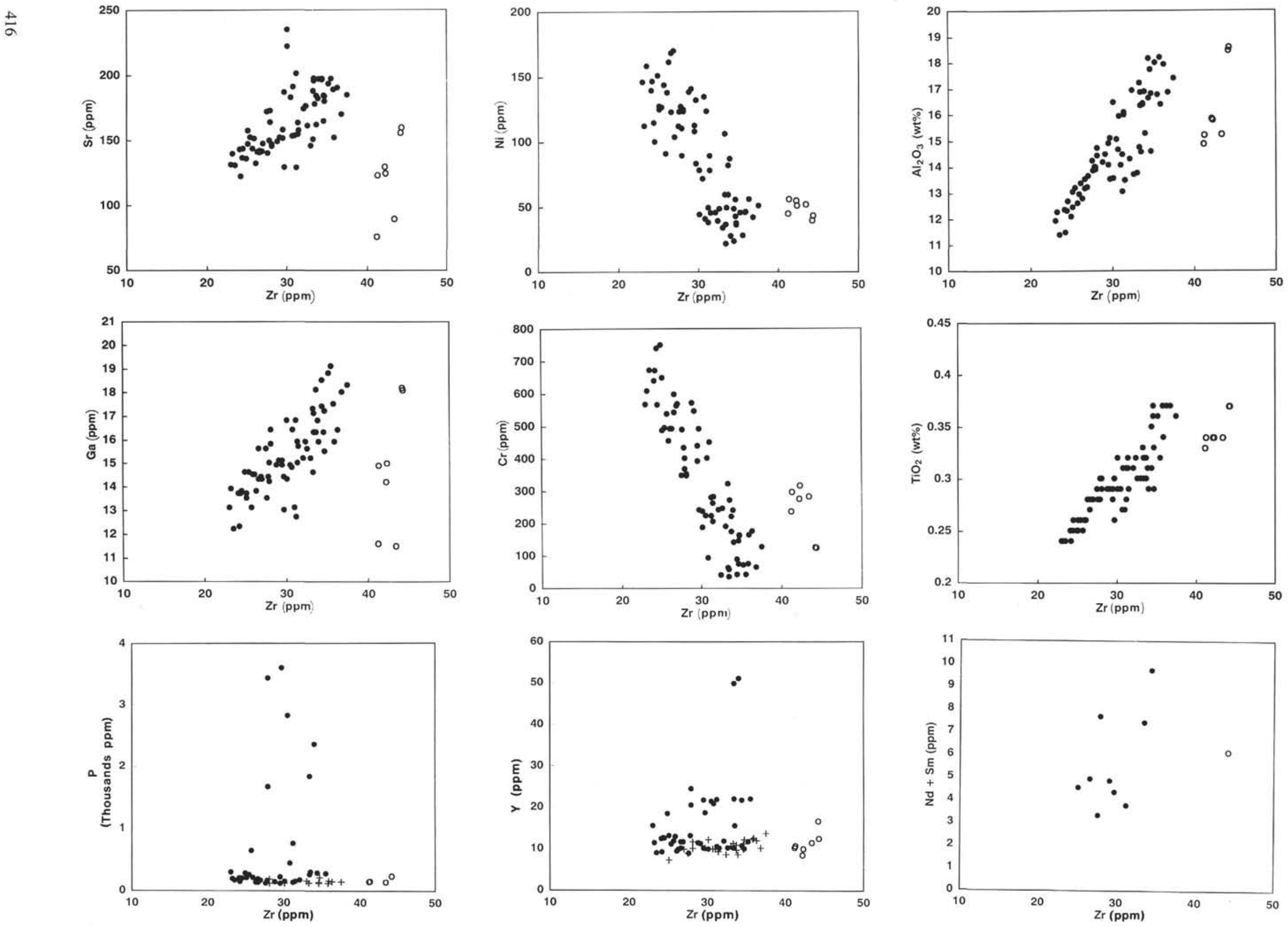

Figure 6. Trace elements (ppm) and $\mathrm{TiO}_{2}$ and $\mathrm{Al}_{2} \mathrm{O}_{3}(\mathrm{wt} \%)$ plotted vs. $\mathrm{Zr}(\mathrm{ppm})$ for Hole 793B basement. Open circles $=\mathrm{Group} \mathrm{A}$ lavas, and filled circles $=\mathrm{Group} \mathrm{B}$ lavas. 


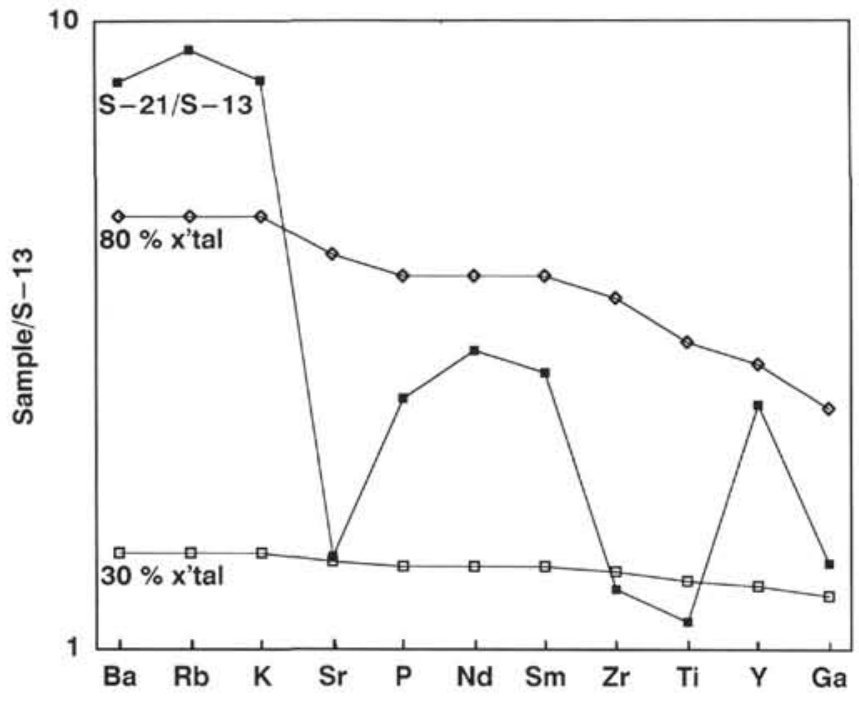

Figure 7. Trace element concentration of Sample S-21 (3.24 wt\% $\mathrm{MgO}$ ) normalized to Sample S-13 (11.58 wt\% MgO). Also shown are predicted profiles for magmas generated by $30 \%$ and $80 \%$ crystallization (x'tal) of Sample S-13. Crystallization modeled using distribution coefficients from Henderson (1982) and phase proportions cpx:opx:plag $=5: 4: 1$.

appears to be commensurate with crystal fractionation/accumulation processes. This can be ascribed to the locking of these elements in the saponite/celadonite/illite alteration products that replace the interstitial glass (for details of clay chemistry, see Lapierre et al., this volume).

Interestingly, $\mathrm{Sr}$, which is normally considered to be mobile, shows a variation within the sequence commensurate with primary igneous fractionation. This $\mathrm{Sr}$ behavior can be explained by its location within the whole-rock system. All lavas within Hole 793B have abundant plagioclase laths and microlites in their groundmass. Strontium would behave incompatibly during ol-opx-cpx crystallization, concentrating in the liquid with $\mathrm{Zr}, \mathrm{Ti}, \mathrm{P}$, and the REE. However, during final solidification, Sr would partition strongly into the plagioclase microphenocrysts, leaving the incompatible elements in the interstitial glass. During alteration the glass underwent smecti-

Table 4. Rare earth element analyses, Hole 793B basement.

$\begin{array}{rrrr}\begin{array}{r}\text { Sample } \\ \text { Core, section }\end{array} & \begin{array}{r}\mathrm{S}-12 \\ 96 \mathrm{R}-1\end{array} & \begin{array}{r}\mathrm{S}-20 \\ 112 \mathrm{R}-1\end{array} \\ \begin{array}{r}\text { Interval (cm) } \\ \text { Unit }\end{array} & 41-83 \mathrm{~cm} & 59-63 \mathrm{~cm} & \\ & 4 & 14 a & \begin{array}{r}\text { Detection } \\ \text { limits }\end{array} \\ \mathrm{La} & 1.89 & & \\ \mathrm{Ce} & 4.95 & 5.51 & 0.1 \\ \mathrm{Nd} & 3.63 & 3.28 & 0.1 \\ \mathrm{Sm} & 1.20 & 1.04 & 0.015 \\ \mathrm{Eu} & 0.42 & 0.39 & 0.015 \\ \mathrm{Gd} & 1.57 & 1.52 & 0.2 \\ \mathrm{Dy} & 1.54 & 1.78 & 0.02 \\ \mathrm{Er} & 0.99 & 1.33 & 0.02 \\ \mathrm{Yb} & 1.03 & 1.52 & 0.02 \\ \mathrm{Lu} & 0.18 & 0.28 & 0.015 \\ & & & \end{array}$

All concentrations in parts per million. Analysis by isotope dilution mass spectrometry using the technique described by Croudace and Marshall (1991), at the Department of Geology, University of Southampton, U.K.

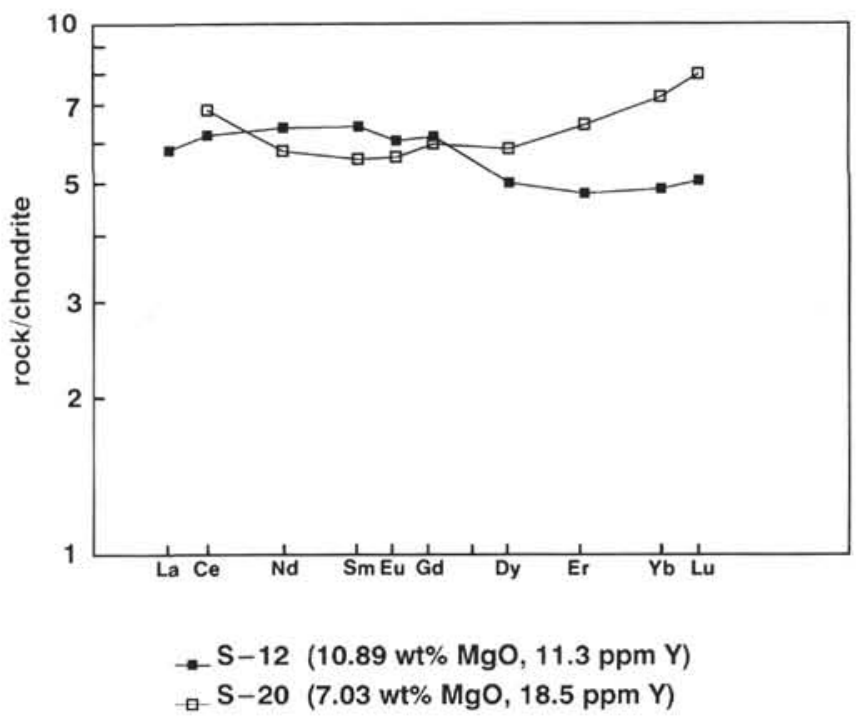

Figure 8. Chondrite-normalized, rare-earth element (REE) profiles for Hole 793B basement lavas. Chondrite values from Kay and Gast (1973).

tization (and possibly illitization) causing partitioning of REE, $P$, and $\mathrm{Y}$ into circulating fluids and leaving hydrolyzed $\mathrm{Ti}$ and $\mathrm{Zr}$ in or around clays, and the $\mathrm{Sr}$ locked in plagioclase. $\mathrm{Sr}$ isotope results (see below) clearly indicate that a minor isotopic exchange between rock and seawater-based fluids has taken place. However, strontium concentrations remain close to the original magmatic abundances.

\section{Forearc Diabase Intrusive}

A diabase sill intruding 14-Ma sediments was encountered at a depth of $586 \mathrm{~m}$ in Hole 793B. Petrographically, the intrusion contains phenocrysts of clinopyroxene, orthopyroxene, and plagioclase and pseudomorphs of olivine. Titanomagnetite was found throughout the intrusion, but it is restricted to the groundmass. Further details of the internal structure and petrography are given in Taylor, Fujioka, et al. (1990) and of the mineral chemistry in Lapierre et al. (this volume).

Major and trace element geochemistry of the intrusion (Table 5) indicates a tholeiitic affinity. Trace element ratios such as $\mathrm{Ti} / \mathrm{Zr}(100)$, $\mathrm{Zr} / \mathrm{Y}(2)$, and $\mathrm{Ti} / \mathrm{V}(12)$ of the diabase are similar to those of the active Izu arc volcanoes of Tori Shima and Sumisu Jima (Shipboard Scientific Party, 1990). A tholeiitic basalt clast derived from the active arc was found in early to middle Miocene sediments $(610 \mathrm{mbsf})$ of Hole 793B. This clast has a similar composition (Sample 012, Table 5) to the diabase, supporting the association of the sill with the active arc.

Laterally extensive seismic reflectors were found across the forearc region (B. Taylor, pers. comm., 1990), which may represent intrusions such as the diabase. In light of these reflectors and the diabase chemistry, it is likely that the intrusion represents a continuation of arc magmatism into the forearc region, or the lateral injection of magmas from the active arc into forearc sediments.

\section{SITE 792}

\section{The Oligocene Arc}

Multichannel seismic profiles across the Izu-Bonin arc have shown the presence of a series of basement promontories, spaced about every $60-100 \mathrm{~km}$, and located $30-40 \mathrm{~km}$ east (trenchward) of the active arc chain (Taylor et al., 1990). These frontal-arc basement highs were recognized by Honza and Tamaki (1985), who named them the Shinkurose Ridge. Initial interpretations designated the ridge as the remnants of an earlier arc-volcano chain, now buried by sediments during the formation of the forearc basin. Hole 792E drilled through 

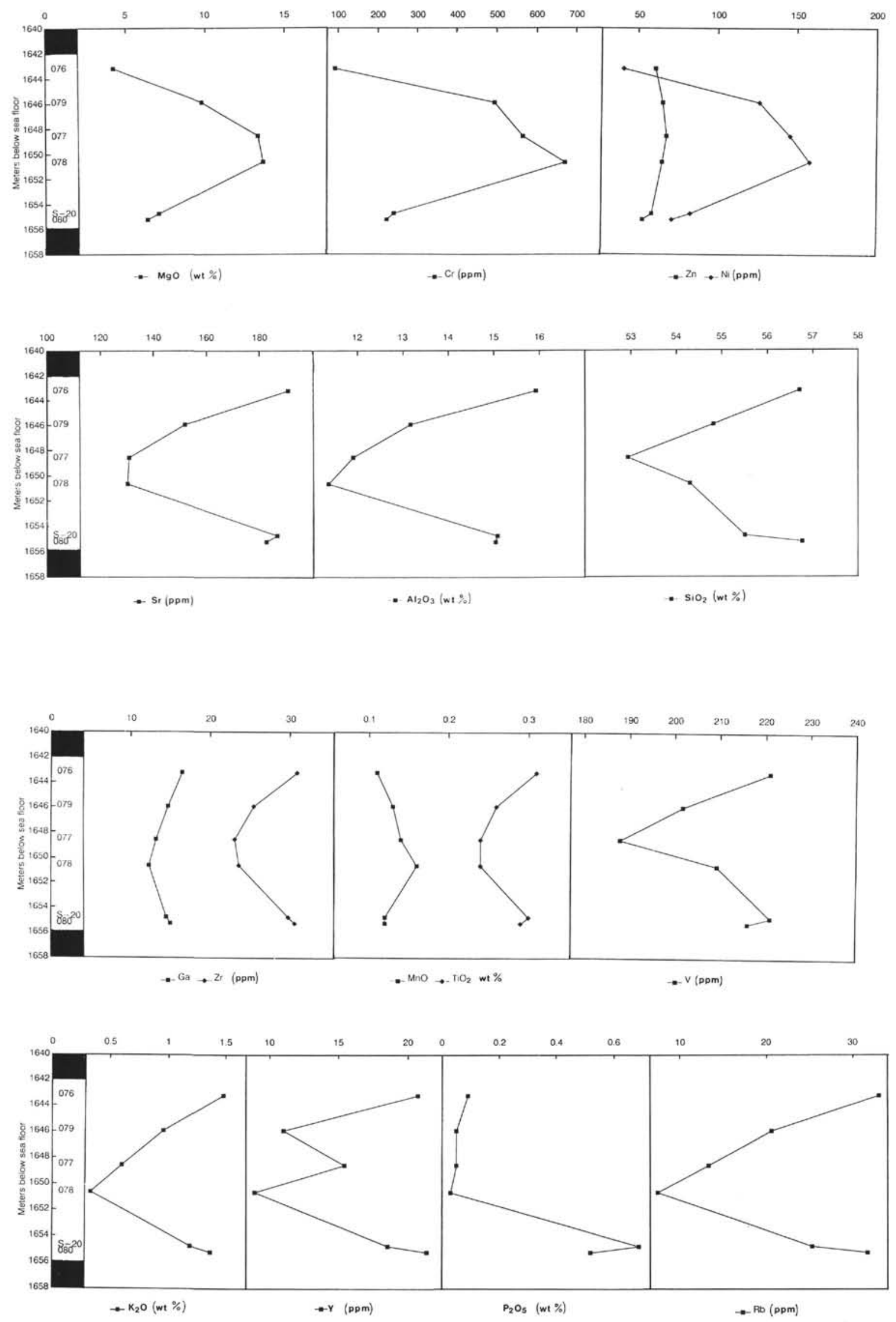

Figure 9. Geochemical profiles through Subunit 14a, Hole 793B basement. Sample numbers used are shown on the left. 
the marginal sediments of the basin and penetrated $82 \mathrm{~m}$ of basement volcanics on a basement high at $32^{\circ} 24^{\prime} \mathrm{N}$ (Fig. 2). The sediments overlying basement were constrained biostratigraphically and magnetostratigraphically as late Oligocene (30-32 Ma), whereas basement volcanics were radiometrically dated as $33 \mathrm{Ma}$. Details of the materials used, analytical procedures, and interpretation of the radiometric ages are given in Taylor and Mitchell (this volume).

Sediments in the $10 \mathrm{~m}$ directly overlying basement are hydrothermally altered volcaniclastic sandstones and conglomerates with clasts of andesite, claystone, and extremely fresh amphibole crystals. The matrix is dominantly blue-grey smectite, replacing original vitric detritus. These sediments suggest that post-eruptive hydrothermal circulation above the basement was concentrated in a restricted horizon. Alteration was probably induced by low-temperature $\left(<200^{\circ} \mathrm{C}\right)$ porewater circulation, in a similar fashion to the vitric-sediment/porewater interaction envisaged for the overlying sediments by Egeberg (1990). Initial studies of the basement lavas indicate a similarly low-temperature alteration with partial replacement of some original phases by smectite, zeolite, and carbonate.

\section{Volcanic Stratigraphy and Eruptive Setting}

Basement volcanics in Hole 792E are subdivided into five major units (Fig. 10). The dominant lithology is massive flows of twopyroxene andesite (estimated at 5-10 m thickness Taylor, Fujioka, et al., 1990) with subordinate dacite, rhyolite, and basaltic andesite. These are intercalated with hyaloclastite breccia layers containing similar mineral species and proportions to the massive flows, but they have matrices of smectitized vitric shards. A single heterolithic volcanogenic breccia is found in the volcanic sequence (Unit 4). This contains subrounded clasts similar to the surrounding flows and a single clast of prehnite-chlorite-bearing meta-basalt. Detailed descriptions of the lithologic units are given in Taylor, Fujioka, et al. (1990).

The combination of hyaloclastitic layers and a heterolithic sedimentary horizon suggests that the volcanics were the products of submarine eruptions. This is consistent with a number of scenarios, any or all of which are plausible: (1) that the volcanic edifice was never emergent above sea level, either because of arc-rifting during construction, or cessation of arc volcanoes, (2) that Site 792 was located on the submerged flank of an arc volcano, and/or (3) that the original volcano has been denuded to its submarine base by subsequent erosion.

\section{Petrography}

Basement volcanics within Hole 792E are petrographically similar to many calc-alkaline lavas in the active Japan Arc. Plagioclase is an abundant and ubiquitous phenocryst (20-30 modal\%) throughout the sequence; it is found associated with two pyroxenes: titanomagnetite and sometimes quartz (Plate 2). The crystallization sequence of plag + oxide $\rightarrow$ opx $+\operatorname{cpx} \rightarrow$ quartz is also typically calc-alkaline. Modal analyses of the lavas are given in Table 6. Quartz is found in all lavas from the lowest $55 \mathrm{~m}$ of the stratigraphy (Fig. 10), regardless of their wholerock $\mathrm{SiO}_{2}$ contents. A brief outline of the mineralogy is given below; a more detailed analysis is given in Lapierre et al. (this volume).

\section{Magnetite}

Subhedral grains $0.3-2 \mathrm{~mm}$ in size, existing as phenocrysts in the groundmass and as inclusions within clino- and orthopyroxene.

\section{Plagioclase}

Euhedral to subhedral fresh phenocrysts $0.5-5 \mathrm{~mm}$ in size. Strong oscillatory zoning is common, with compositions ranging from $\mathrm{An}_{64}$ to $\mathrm{An}_{84}$. Fluid and glass inclusions are abundant, but are restricted to a growth zone between core and rim.

\section{Clinopyroxene}

Euhedral to subhedral unaltered phenocrysts $0.3-3 \mathrm{~mm}$ in size. Zoning is prevalent in most crystals with compositions $\mathrm{En}_{49}: \mathrm{Wo}_{40}$ : $\mathrm{Fs}_{11}-\mathrm{En}_{38}: \mathrm{Wo}_{43}: \mathrm{Fs}_{19}$. Magnetite and glass are found as inclusions in the larger phenocrysts.

\section{Orthopyroxene}

Subhedral phenocrysts $0.3-3 \mathrm{~mm}$ in size are found throughout the sequence; however, with the exception of the uppermost $15 \mathrm{~m}$ of the stratigraphy, are pseudomorphed by clays. The orthopyroxene in the upper flows of Unit 1 have fresh cores mantled by alteration zones comprising a mixture of smectite and cristobalite.

\section{Quartz}

Occurs as subrounded to rounded euhedral grains $<0.1-0.7 \mathrm{~mm}$ in size.

\section{Geochemistry}

We analyzed a series of 26 basement volcanics from Hole 792E to constrain the geochemical characteristics of the Oligocene IzuBonin arc. Major and trace element data are presented in Table 7. The major element data reveal a diverse compositional range within the $82 \mathrm{~m}$ of basement drilled. Samples range from basaltic andesites, through andesites and dacites, to rhyolite (about 53-72 wt $\% \mathrm{SiO}_{2}$ and $1-7 \mathrm{wt} \% \mathrm{MgO}$ ). Evolutionary trends within the major element data (Fig. 11) are typical of calc-alkaline suites, with a decline in total iron and a steady increase in $\mathrm{SiO}_{2}$ with decreasing $\mathrm{MgO}$ contents.

As the basement lavas are to some extent altered, it is important to assess how secondary processes have affected original chemical compositions. Petrographically, the freshest samples are in the upper $20 \mathrm{~m}$ of Unit 1 and the most altered lavas are found in Units 3-5. The consistency of most immobile elements within the various flows of Unit 1 also suggests that these andesites are close to their unaltered compositions. The contrast between the upper and lower units of the section is paralleled by chemostratigraphic variations, particularly in the distribution of $\mathrm{MgO}$, as shown in Figure 10. Units 3-5 are intercalations of basaltic andesite, andesite, and dacite/rhyolite whereas Units 1 and 2 are entirely andesite. It is, therefore, a possibility that the extremes found in the lower units are simply a function of an alteration process. Support for this comes from observations that the basaltic andesites are fractured and friable, suggesting a fluid-related density decrease, whereas the rhyolitic rocks are hard and have silicified groundmasses, commensurate with bulk silica addition.

Lavas from Hole 792E show similarly anomalous trace element features to the forearc basin basement of Hole 793B. Notably, Hole 792E lavas show large variations in P and Y (Fig. 12) not commensurate with the quantities of crystallization estimated from major element compositions. This is investigated further by comparing average analyses of basaltic andesites, andesites, and dacites. Figure 13 shows the relative enrichment/depletion of trace elements in average basaltic andesite and dacite relative to average andesite. Contrary to normal fractionation systems, the basaltic andesite has higher $\mathrm{Zr}$ and lower $\mathrm{Ni}$ and $\mathrm{Cr}$ compared with average andesite. Furthermore, the basaltic andesite has a factor of $2-3$ less $\mathrm{P}, \mathrm{Y}, \mathrm{Rb}, \mathrm{K}$, and $\mathrm{Ba}$. The average dacite could be derived from the andesite by fractional crystallization in light of the lower $\mathrm{Ni}$ and $\mathrm{Cr}$. However, the dacite profile in Figure 13 has similar levels of Ti, $\mathrm{Zr}$, and $\mathrm{Sr}$ to the basaltic andesite, combined with a relative enrichment of $P, Y$, and the LFS elements.

The basaltic andesites have similar phenocryst mineral proportions to the andesites, with the exception of the andesites at the top that lack quartz. As the basaltic andesites are more altered than the andesites, the possibility is raised that the basaltic andesites are simply 
Table 5. Diabase sill and basalt pebble geochemistry, Hole 793B.

\begin{tabular}{|c|c|c|c|c|c|c|c|c|c|c|c|c|c|}
\hline Core, section & $1 R-1$ & $1 \mathrm{R}-1$ & $1 R-1$ & $1 R-1$ & $1 R-2$ & $1 R-2$ & $1 R-2$ & $1 \mathrm{R}-2$ & $1 \mathrm{R}-3$ & $1 R-3$ & $1 R-3$ & $3 R-1$ & $3 R-1$ \\
\hline Interval & $1 / 35-39$ & $1 / 73-77$ & $1 / 115-119$ & $1 / 132-136$ & $2 / 15-19$ & $2 / 25-31$ & $2 / 61-65$ & $2 / 91-95$ & $3 / 1-5$ & $3 / 27-31$ & $3 / 77-82$ & $1 / 95-98$ & $1 / 98-100$ \\
\hline Depth (MBSF) & 586.79 & 587.23 & 587.65 & 587.82 & 588.15 & 588.27 & 588.60 & 588.92 & 589.36 & 589.58 & 590.12 & 605.00 & 605.28 \\
\hline Sample no. & 001 & 002 & 003 & 004 & 005 & 006 & 007 & 008 & 009 & 010 & 011 & $s-1$ & 012 \\
\hline & 1 & 1 & I & 1 & 1 & 1 & I & 1 & 1 & 1 & 1 & 1 & I \\
\hline Lithology & Diabase & Diabase & Diabase & Diabase & Diabase & Diabase & Diabase & Diabase & Diabase & Diabase & Diabase & Basalt & Basalt \\
\hline \multicolumn{14}{|c|}{ Major elements (wt\%) } \\
\hline $\mathrm{SiO} 2$ & 52.84 & 52.52 & 52.65 & 52.07 & 52.24 & 52.61 & 53.10 & 52.87 & 52.99 & 53.05 & 52.58 & 52.81 & 52.68 \\
\hline $\mathrm{TiO} 2$ & 0.58 & 0.52 & 0.57 & 0.53 & 0.53 & 0.58 & 0.58 & 0.56 & 0.59 & 0.61 & 0.56 & 0.66 & 0.65 \\
\hline $\mathrm{Al} 2 \mathrm{O} 3$ & 14.85 & 14.70 & 14.44 & 14.46 & 14.37 & 14.51 & 14.68 & 14.80 & 14.86 & 14.71 & 14.63 & 17.07 & 16.76 \\
\hline $\mathrm{Fe} 2 \mathrm{O} 3$ * & 10.61 & 9.68 & 10.34 & 10.03 & 10.17 & 10.44 & 9.80 & 9.82 & 10.05 & 10.05 & 9.65 & 10.42 & 10.24 \\
\hline $\mathrm{MnO}$ & 0.16 & 0.17 & 0.19 & 0.19 & 0.19 & 0.19 & 0.16 & 0.15 & 0.16 & 0.16 & 0.17 & 0.16 & 0.18 \\
\hline $\mathrm{MgO}$ & 8.56 & 9.34 & 8.95 & 9.41 & 9.68 & 9.10 & 8.99 & 8.80 & 8.67 & 8.72 & 9.26 & 5.27 & 5.33 \\
\hline $\mathrm{CaO}$ & 10.19 & 10.85 & 10.82 & 11.01 & 10.94 & 10.80 & 10.49 & 10.27 & 10.39 & 10.28 & 10.76 & 11.70 & 11.61 \\
\hline $\mathrm{Na} 2 \mathrm{O}$ & 1.94 & 1.86 & 1.72 & 1.64 & 1.63 & 1.70 & 1.88 & 1.97 & 1.93 & 2.03 & 1.96 & 1.91 & 2.13 \\
\hline K2O & 0.26 & 0.11 & 0.27 & 0.26 & 0.20 & 0.18 & 0.32 & 0.32 & 0.36 & 0.34 & 0.28 & 0.30 & 0.34 \\
\hline $\mathrm{P} 2 \mathrm{O} 5$ & 0.06 & 0.05 & 0.05 & 0.05 & 0.05 & 0.05 & 0.06 & 0.05 & 0.05 & 0.06 & 0.06 & 0.04 & 0.06 \\
\hline (LOI) & 0.48 & 0.37 & 0.20 & 0.55 & 0.46 & 0.33 & 0.30 & 0.53 & 0.25 & 0.36 & 0.68 & 0.33 & 0.00 \\
\hline Total & 100.05 & 99.80 & 100.00 & 99.65 & 100.00 & 100.16 & 100.06 & 99.61 & 100.05 & 100.01 & 99.91 & 100.34 & 99.98 \\
\hline \multicolumn{14}{|c|}{ Trace elements (ppm) } \\
\hline P & 281 & 287 & 261 & 249 & 258 & 282 & 296 & 273 & 293 & 303 & 278 & & 179 \\
\hline $\mathrm{Zr}$ & 34.4 & 32.8 & 35.2 & 32.6 & 31.9 & 35.0 & 36.4 & 35.3 & 35.5 & 38.6 & 34.2 & & 37.6 \\
\hline$Y$ & 17.9 & 17.2 & 18.4 & 17.4 & 17.2 & 18.4 & 18.1 & 17.3 & 17.9 & 17.7 & 17.7 & & 21.1 \\
\hline $\mathrm{Ga}$ & 14.9 & 15.4 & 14.7 & 14.9 & 14.2 & 15.1 & 14.9 & 14.8 & 14.8 & 15.1 & 14.4 & & 15.9 \\
\hline $\mathrm{Rb}$ & 2.8 & 1.8 & 3.6 & 4.1 & 3.6 & 2.9 & 4.3 & 4.2 & 5.8 & 5.5 & 4.8 & & 5.1 \\
\hline $\mathrm{Sr}$ & 116 & 119 & 116 & 113 & 112 & 117 & 116 & 117 & 117 & 116 & 114 & & 140 \\
\hline $\mathrm{Ba}$ & 37 & 52 & 103 & 45 & 46 & 64 & 66 & 55 & 67 & 50 & 45 & & 32 \\
\hline V & 288 & 252 & 284 & 266 & 261 & 280 & 277 & 265 & 280 & 282 & 290 & & 323 \\
\hline $\mathrm{Nb}$ & 0.4 & 0.0 & 0.0 & 0.0 & 0.0 & 0.5 & 0.0 & 0.1 & 0.3 & 0.7 & 0.3 & & 0.4 \\
\hline $\mathrm{Ni}$ & 76 & 99 & 93 & 104 & 113 & 97 & 100 & 89 & 92 & 91 & 106 & & 25 \\
\hline $\mathrm{Cr}$ & 167 & 252 & 225 & 260 & 249 & 233 & 255 & 200 & 220 & 224 & 275 & & 102 \\
\hline $\mathrm{Zn}$ & 69 & 66 & 72 & 70 & 70 & 71 & 73 & 67 & 72 & 72 & 72 & & 79 \\
\hline $\mathrm{Cu}$ & 58 & 84 & 109 & 112 & 84 & 93 & 99 & 97 & 95 & 105 & 88 & & 90 \\
\hline \multirow{2}{*}{\multicolumn{14}{|c|}{$\begin{array}{l}\mathrm{Nd} \\
\mathrm{Sm}\end{array}$}} \\
\hline & & & & & & & & & & & & & \\
\hline $100 \mathrm{Mg} / \mathrm{Mg}+\mathrm{Fe}(\mathrm{t})$ & 64.0 & 68.0 & 65.6 & 67.4 & 67.7 & 65.7 & 66.9 & 66.4 & 65.5 & 65.6 & 67.9 & 52.7 & 53.4 \\
\hline $\mathrm{Al} 2 \mathrm{O} 3 / \mathrm{TiO} 2$ & 25.6 & 28.3 & 25.3 & 27.3 & 27.1 & 25.0 & 25.3 & 26.4 & 25.2 & 24.1 & 26.1 & 25.9 & 25.8 \\
\hline $\mathrm{CaO} / \mathrm{TiO} 2$ & 17.6 & 20.9 & 19.0 & 20.8 & 20.6 & 18.6 & 18.1 & 18.3 & 17.6 & 16.9 & 19.2 & 17.7 & 17.9 \\
\hline $\mathrm{CaO} / \mathrm{Al} 2 \mathrm{O} 3$ & 0.69 & 0.74 & 0.75 & 0.76 & 0.76 & 0.74 & 0.71 & 0.69 & 0.70 & 0.70 & 0.74 & 0.69 & 0.69 \\
\hline $\mathrm{Ti} / \mathrm{Zr}$ & 101 & 95 & 97 & 97 & 100 & 99 & 96 & 95 & 100 & 95 & 98 & & 104 \\
\hline $\mathrm{Zr} / \mathrm{Y}$ & 1.9 & 1.9 & 1.9 & 1.9 & 1.9 & 1.9 & 2.0 & 2.0 & 2.0 & 2.2 & 1.9 & & 1.8 \\
\hline
\end{tabular}

Major elements (wt\%) 


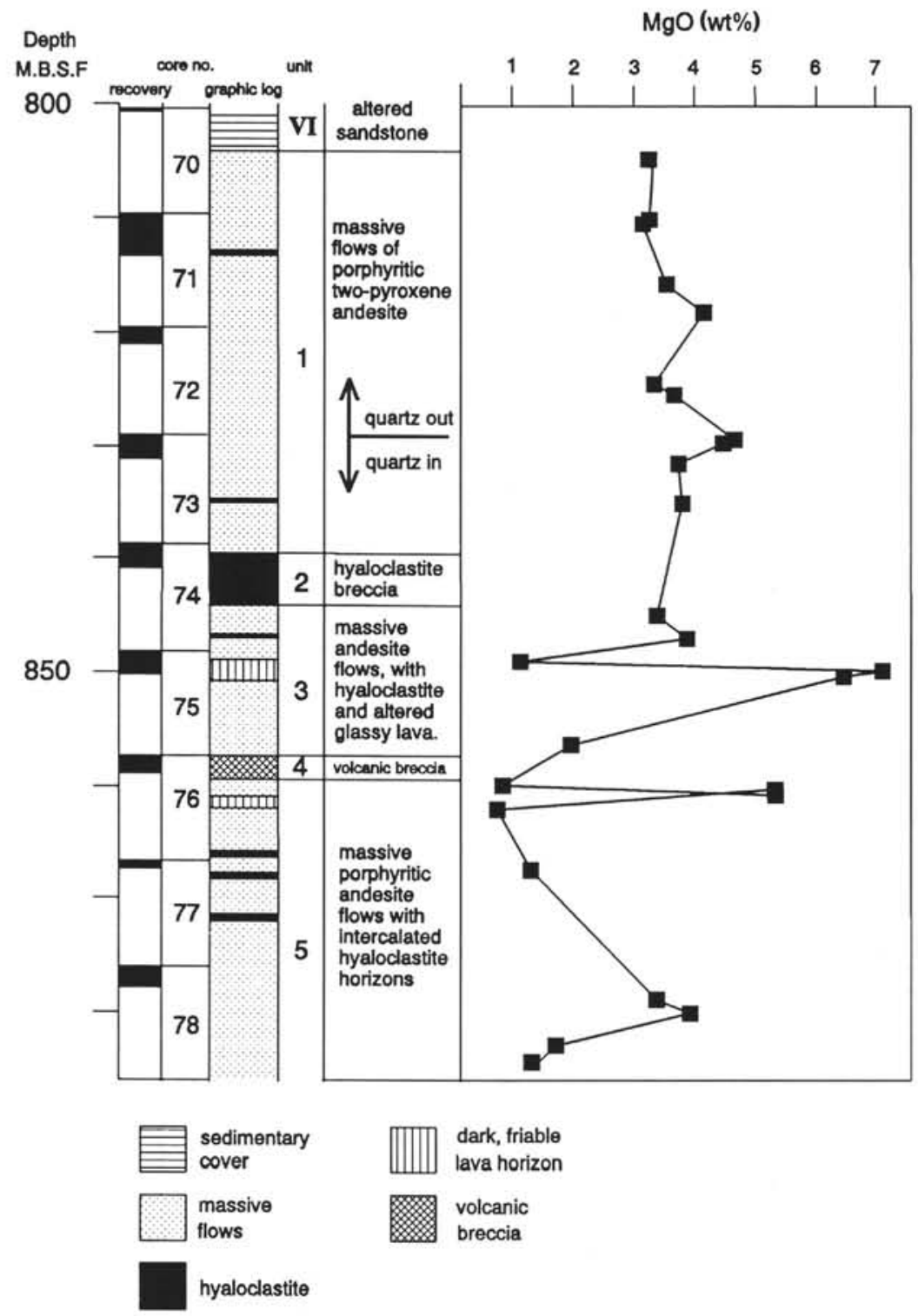

Figure 10. Stratigraphic summary of basement rock sequence in Hole 792E, Cores 126-792E-70R through $-78 \mathrm{R}$. Recovery within each core is proportionally expanded to fill the total core penetration. $\mathrm{MgO}$ values are plotted vs. depth for all samples analyzed from coherent igneous units.

andesites that have undergone hydrothermal alteration. The net result of the alteration is a decrease in silica, P, Y, and LFS elements with a resultant increase in the contents of the immobile elements $\mathrm{Ti}$ and $\mathrm{Zr}$. Rhyolite and, to a limited extent, dacite compositions are likely to be the result of net deposition of the solute elements along particular horizons. As with the Hole 793B basement, $\mathrm{Sr}$ is apparently relatively stable during the leaching process and can again be explained by the retention of $\mathrm{Sr}$ in unaltered plagioclase.

\section{RADIOGENIC ISOTOPE GEOCHEMISTRY OF FOREARC VOLCANICS}

Basement volcanics from Holes 793B and 792E together with a sample from the Neogene intrusion in Hole 793B were analyzed for $\mathrm{Sr}, \mathrm{Nd}$, and $\mathrm{Pb}$ isotopes. Lead isotopes were analyzed at Royal Holloway and Bedford New College, London, using a VG354 fivecollector mass-spectrometer with static data collection. Ratios were normalized for mass fractionation to SRM981 by about $0.11 \% / a m u$.
Internal errors are estimated at better than $0.005 \%$ /amu and reproducibility at better than $0.05 \% / \mathrm{amu}$. $\mathrm{Sr}$ and $\mathrm{Nd}$ isotopes were measured at Universite Blaise Pascal, Clermont-Ferrand, France. Pb, Nd, and $\mathrm{Sr}$ isotope data are presented in Table 8.

\section{Sr-Nd Isotopes}

The nine samples from Hole 793B basement have consistent ${ }^{143} \mathrm{Nd} /{ }^{144} \mathrm{Nd}$ ratios (Fig. 14), with $\varepsilon_{\mathrm{Nd}}$ ranging from 5.63 to $6.82(\mathrm{t}=$ $30 \mathrm{Ma}) .87 \mathrm{Sr} /{ }^{86} \mathrm{Sr}$ ratios are slightly more dispersed $(0.7038-0.7044)$ indicating that basalt-seawater interaction or hydrothermal fluids have disturbed the original proportions of radiogenic Sr. However, ${ }^{143} \mathrm{Nd} /{ }^{144} \mathrm{Nd}$ ratios do not correlate with enrichment of the LFS elements or the unusual $\mathrm{P}$ and $\mathrm{Y}$ variations. This suggests that despite the apparent mobility of the REE during basement alteration, the fluids were of a similar Nd-isotopic composition to the lavas. As such, we propose that the fluids causing the redistribution of the REE obtained their REE solute from within the lava pile. If the fluids did 
Table 6. Modal mineralogy of basement rocks, Hole 792E.

\begin{tabular}{llllllll}
\hline $\begin{array}{l}\text { Sample/ } \\
\text { analysis }\end{array}$ & $\begin{array}{c}\text { Core, section, } \\
\text { interval }(\mathrm{cm})\end{array}$ & $\begin{array}{c}\text { Depth } \\
\text { (mbsf) }\end{array}$ & Plag & Opx & Cpx & Qtz & Opaques \\
\hline A - & $71 \mathrm{R}-\mathrm{CC}, 10$ & 812.6 & 36.5 & 5.0 & 3.5 & - & 1.7 \\
B & $74 \mathrm{R}-1,146$ & 840.3 & 23.1 & 0.1 & 6.3 & - & 8.9 \\
C & $74 \mathrm{R}-1,146$ & 840.3 & 30.7 & 5.9 & 1.8 & - & 2.4 \\
D S31 & $76 \mathrm{R}-1,61$ & 858.3 & 35.5 & 6.5 & 1.2 & 1.5 & 1.2 \\
E S-34 & $78 \mathrm{R}-1,134$ & 877.9 & 32.8 & 6.5 & 4.4 & 1.3 & 1.3 \\
\hline
\end{tabular}

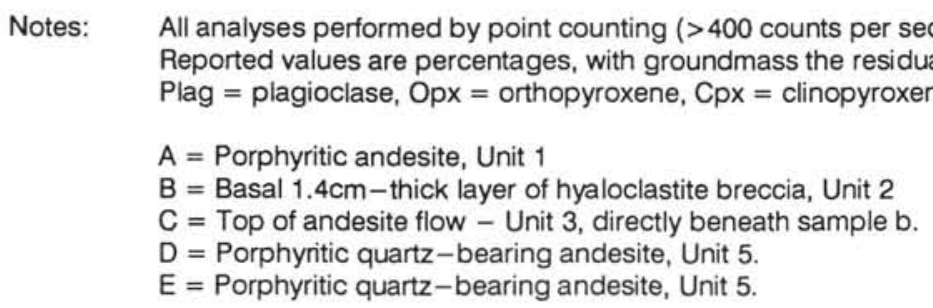

circulate through sediments or lower regions of the basement, either they did not scavenge significant quantities of REE in these locations, or these lithologies had similar ${ }^{143} \mathrm{Nd} /{ }^{144} \mathrm{Nd}$ systematics.

In comparison to other lavas in Izu-Bonin arc-trench system (Fig. 14), the Hole 793B basement has lower $\varepsilon_{\mathrm{Nd}}$ than MORB and the active arc, and it lies at the upper end of the spectrum of boninites from the Izu-Bonin outer-arc high.

\section{Pb Isotopes}

Lead isotopes were measured on six samples from Hole 793B basement, three samples from Hole 792E, and one sample from the Neogene diabase sill in Hole 793B. Figures 15 and 16 compare ${ }^{207 / 204} \mathrm{~Pb}$ ${ }^{206 / 204} \mathrm{~Pb}$ and ${ }^{208 / 204} \mathrm{~Pb}^{-206 / 204} \mathrm{~Pb}$ data from Leg 126 with analyses from potential components and lavas from the western Pacific environs. Lavas from the forearc basement at Sites 792 and 793 lie within the range of isotopic compositions of Pacific MORB, on or slightly above the Northern Hemisphere reference line ( $\Delta 7 / 4$ and $\Delta 8 / 4$ values range from -1.74 to 3.3 and 7.3 to 37.4 , respectively). The diabase sill lies between the Pacific MORB and western Pacific sediment fields on both ${ }^{207 / 204} \mathrm{~Pb}^{-206 / 204} \mathrm{~Pb}$ and ${ }^{208 / 204} \mathrm{~Pb}-{ }^{206 / 204} \mathrm{~Pb}$ plots $(\Delta 7 / 4$ and $\Delta 8 / 4$ are 7.2 and 52.4 , respectively).

Unlike the Bonin Islands (Dobson and Tilton, 1989), no clear evidence for $\mathrm{a}^{207 / 204} \mathrm{~Pb}$-rich sedimentary component was found in the basement lavas of the forearc basin. However, the ${ }^{207 / 204} \mathrm{~Pb}$ datum from the diabase sill (Fig. 15 and 16) suggests that sediments may have played a role in its petrogenesis. Contamination of the sill magma could have taken place either in the mantle source by introduced slab sediments or by digestion of host sediments during intrusion. The lack of available $\mathrm{Pb}$ data for Izu arc lavas of comparable composition as well as intruded sedimentary horizons precludes an interpretation regarding the nature of contamination.

\section{PETROGENETIC RELATIONS BETWEEN ARC-TRENCH MAGMAS}

The occurrence of boninite series volcanics (BSV) in the forearc terrains of the Western Pacific has been well documented (e.g., Dietrich et al., 1978; Hickey and Frey, 1982; Umino, 1986; Bloomer and Hawkins, 1987). However, the question remains as to whether the current location of boninite lavas bears any relation to their original position with respect to the subduction zone and arc. Drilling during Leg 126 sampled igneous units within the forearc region which, combined with age constraints, suggest that the boninites were generated within current tectonic framework.

The Hole 793B diabase sill is younger than about $12 \mathrm{Ma}$ and was recovered from the center of the forearc basin. However, its similar composition to Torishima and other Izu arc volcanoes, together with the possibility of lateral injection from the arc, do not allow this intrusion to be constrained as forearc volcanism. Age relations between basement at Sites 792 and 793 do provide an insight into the temporal development of the arc-trench crust. The calc-alkaline volcanic edifice drilled at Site 792, combined with the seismic evidence, demonstrates that during the Oligocene (about 30-33 Ma) a well-developed arc-chain existed in a similar orientation to the modern arc. Basement lavas from Hole 793B, trenchward of Site 792, are interpreted as being in the age range from 26 to $33 \mathrm{Ma}$, (Taylor and Mitchell, this volume). These lavas were clearly produced in a forearc environment in response to basin formation between arc and outer-arc high. As such, Site 793B basement represents one of the few wellconstrained examples of forearc volcanism.

Comparable tectonic structures and age relationships along the length of the Izu and Mariana subduction systems suggest that little tectonic erosion has taken place between the outer-arc high and trench in the duration of this subduction system. The absence of thrust-related structures across the arc suggests that tectonic units within the forearc have not been juxtapositioned. Hence, it is likely that the outer-arc high lavas were also generated in a forearc location.

In light of the consistent relative positions between tectonic units, Taylor et al. (1992) examined spatial geochemical variations within the Izu-Bonin arc-trench region. These authors found that indexes of mantle depletion (e.g., $\mathrm{TiO}_{2}, \mathrm{Y}$, and $\mathrm{Al}_{2} \mathrm{O}_{3} / \mathrm{TiO}_{2}$ ) displayed a gradual transition from the arc toward the trench. Figure 17 shows the decline in $\mathrm{TiO}_{2}$ contents of lavas from the backarc to the forearc, reflecting a more depleted mantle source toward the trench. On the active arc, the lowest-Ti lavas are primitive island arc tholeiites, similar to the Hole 793B diabase intrusion (about $0.5 \mathrm{wt} \% \mathrm{TiO}_{2}$ at $9 \mathrm{wt} \% \mathrm{MgO}$ ). In the forearc rift-basin, basement lavas are low-Ti tholeiites transitional to boninites $\left(0.3 \mathrm{wt} \% \mathrm{TiO}_{2}\right.$ at $\left.9 \mathrm{wt} \% \mathrm{MgO}\right)$. Boninitic volcanics closest to the trench have the lowest $\mathrm{TiO}_{2}$ concentrations $(0.15 \mathrm{wt} \%)$. This lateral variation in geochemistry can be related to a compositionally stratified mantle combined with variable melting location in response to slab depth (Taylor et al., 1992).

Lavas from the Izu-Bonin forearc basin are transitional between arc tholeiites and boninites in terms of their petrography and trace element depletion. However, an important char- 

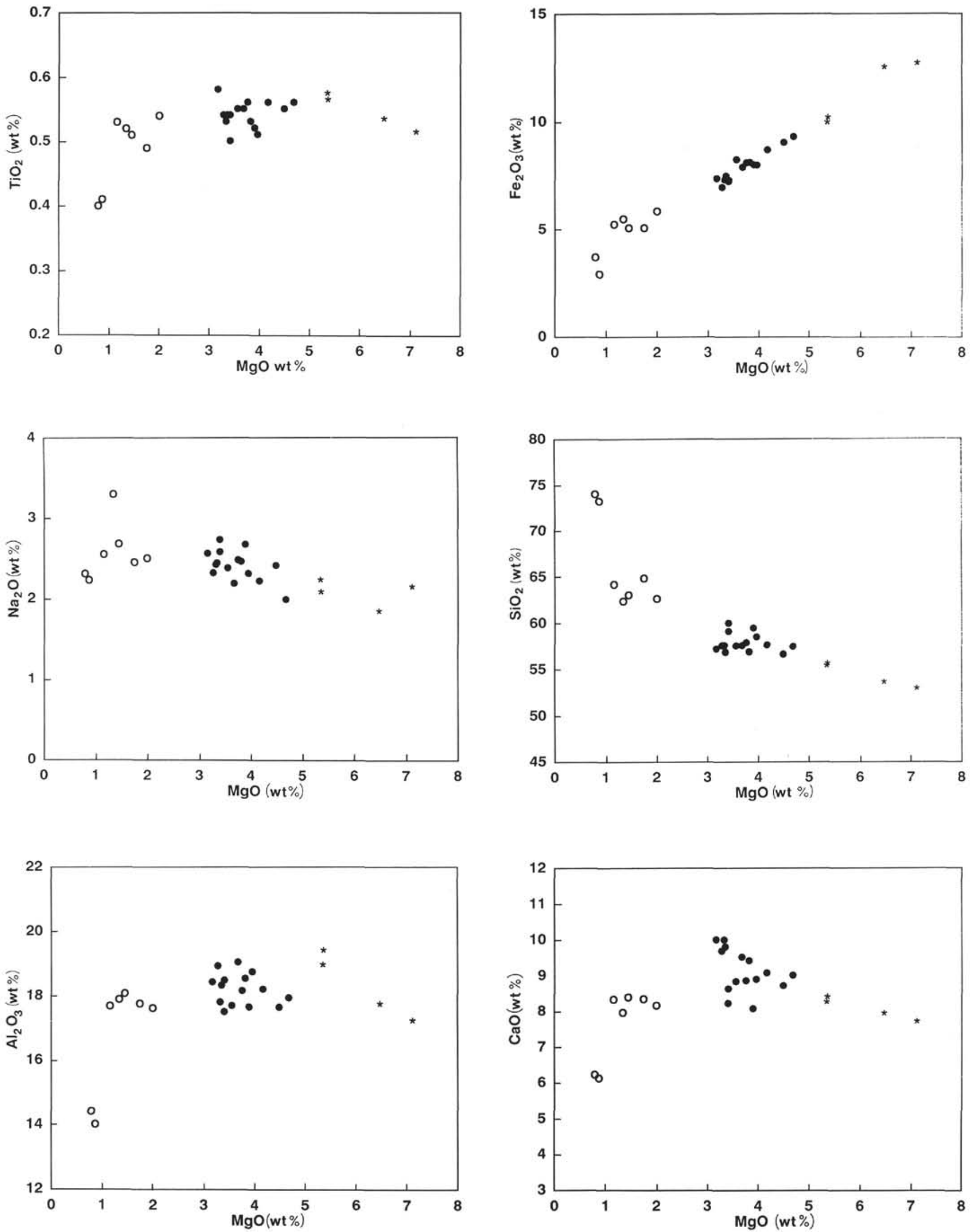

Figure 11. Major elements plotted vs. $\mathrm{MgO}$ for Hole 792E basement (all values in wt $\%$ ). 
Table 7. Basement geochemistry, Hole 792E.

\begin{tabular}{|c|c|c|c|c|c|c|c|c|c|c|c|c|c|c|c|}
\hline Core, section & $70 R-c c$ & $71 \mathrm{R}-1$ & $71 R-1$ & $71 R-2$ & $71 R-3$ & $72 R-1$ & $72 R-1$ & $73 R-1$ & $73 R-1$ & $73 R-1$ & $73 R-2$ & $73 R-2$ & $74 R-2$ & $75 R-1$ & $75 R-1$ \\
\hline Interval & $16-20$ & $9-13$ & $18-21$ & $89-92$ & $34-38$ & $100-104$ & $111-115$ & $15-18$ & $18-21$ & $72-76$ & $3-7$ & $53-57$ & $104-108$ & $18-22$ & $49-51$ \\
\hline Depth (MBSF) & 805.00 & 810.00 & 810.38 & 815.66 & 818.20 & 824.59 & 825.50 & 829.50 & 829.84 & 831.60 & 835.12 & 845.00 & 847.10 & 849.11 & 850.00 \\
\hline Sample no. & 099 & $s-24$ & 100 & 101 & $s-25$ & 103 & $S-26$ & $s-27$ & 104 & 105 & 106 & $S-28$ & 110 & 112 & 113 \\
\hline Unit & 1 & 1 & 1 & 1 & 1 & 1 & 1 & 1 & 1 & 1 & 1 & 3 & 3 & 3 & 3 \\
\hline Lithology & Andesite & Andesite & Andesite & Andesite & Andesite & Andesite & Andesite & Andesite & Andesite & Andesite & Andesite & Andesite & Andesite & Dacite & Bas.and \\
\hline \multicolumn{16}{|c|}{ Major elements (wt\%) } \\
\hline $\mathrm{SiO} 2$ & 57.47 & 57.48 & 57.12 & 57.44 & 57.55 & 56.77 & 57.48 & 57.39 & 56.55 & 57.79 & 56.83 & 59.91 & 59.39 & 64.20 & 52.57 \\
\hline $\mathrm{THO} 2$ & 0.53 & 0.54 & 0.58 & 0.55 & 0.56 & 0.54 & 0.55 & 0.56 & 0.55 & 0.56 & 0.53 & 0.54 & 0.52 & 0.53 & 0.51 \\
\hline $\mathrm{Al} 2 \mathrm{O} 3$ & 17.80 & 18.92 & 18.42 & 17.69 & 18.20 & 18.32 & 19.04 & 17.93 & 17.64 & 18.15 & 18.53 & 17.50 & 17.65 & 17.70 & 17.15 \\
\hline $\mathrm{Fe} 2 \mathrm{O} 3$ * & 7.26 & 6.90 & 7.31 & 8.19 & 8.65 & 7.42 & 7.84 & 9.26 & 8.99 & 8.05 & 8.07 & 7.17 & 7.95 & 5.24 & 12.56 \\
\hline $\mathrm{MnO}$ & 0.31 & 0.15 & 0.25 & 0.32 & 0.14 & 0.13 & 0.08 & 0.10 & 0.13 & 0.13 & 0.18 & 0.05 & 0.09 & 0.09 & 0.17 \\
\hline $\mathrm{MgO}$ & 3.33 & 3.28 & 3.17 & 3.56 & 4.17 & 3.35 & 3.68 & 4.68 & 4.49 & 3.76 & 3.82 & 3.41 & 3.90 & 1.16 & 7.11 \\
\hline $\mathrm{CaO}$ & 9.98 & 9.67 & 9.99 & 8.82 & 9.06 & 9.79 & 9.50 & 8.99 & 8.70 & 8.84 & 9.40 & 8.21 & 8.06 & 8.34 & 7.63 \\
\hline $\mathrm{Na} 2 \mathrm{O}$ & 2.41 & 2.31 & 2.55 & 2.37 & 2.21 & 2.43 & 2.18 & 1.98 & 2.40 & 2.47 & 2.45 & 2.57 & 2.66 & 2.55 & 2.10 \\
\hline K2O & 0.19 & 0.23 & 0.28 & 0.55 & 0.15 & 0.18 & 0.13 & 0.14 & 0.16 & 0.20 & 0.17 & 0.20 & 0.17 & 0.28 & 0.17 \\
\hline $\mathrm{P} 2 \mathrm{O} 5$ & 0.15 & 0.11 & 0.11 & 0.07 & 0.05 & 0.47 & 0.13 & 0.04 & 0.06 & 0.06 & 0.09 & 0.04 & 0.06 & 0.09 & 0.03 \\
\hline (LOI) & 1.77 & 1.01 & 0.96 & 0.93 & 0.81 & 1.26 & 1.05 & 1.10 & 0.98 & 0.53 & 1.30 & 1.44 & 1.17 & 0.72 & 1.71 \\
\hline Total & 99.43 & 99.59 & 99.78 & 99.56 & 100.74 & 99.40 & 100.61 & 101.07 & 99.67 & 100.01 & 100.07 & 99.60 & 100.45 & 100.18 & 100.00 \\
\hline \multicolumn{16}{|c|}{ Trace elements (ppm) } \\
\hline$P$ & 635 & & 444 & 281 & & 2244 & & & 256 & 257 & 376 & & 227 & 355 & 92 \\
\hline $\mathrm{Zr}$ & 39.2 & 39.9 & 41.1 & 39.0 & 39.9 & 40.4 & 42.2 & 41.4 & 41.1 & 41.8 & 41.1 & 38.7 & 41.7 & 41.8 & 45.3 \\
\hline Y & 24.5 & 20.3 & 25.7 & 20.8 & 20.3 & 46.8 & 28.1 & 21.8 & 22.8 & 22.5 & 25.0 & 18.4 & 23.7 & 27.2 & 7.2 \\
\hline $\mathrm{Ga}$ & 16.0 & & 15.8 & 15.8 & & 16.3 & & & 16.0 & 15.8 & 15.8 & & 15.8 & 16.0 & 14.2 \\
\hline $\mathrm{Rb}$ & 3.0 & 1.4 & 3.3 & 10.3 & 1.4 & 3.1 & 1.2 & 1.3 & 3.1 & 3.1 & 3.4 & 2.4 & 3.6 & 4.3 & 2.8 \\
\hline $\mathrm{Sr}$ & 172 & 176 & 175 & 166 & 176 & 185 & 188 & 175 & 170 & 170 & 177 & 166 & 167 & 160 & 180 \\
\hline $8 a$ & 18 & 31 & 26 & 24 & 31 & 24 & 16 & 12 & 19 & 26 & 10 & 28 & 15 & 24 & 391 \\
\hline V & 249 & 258 & 267 & 251 & 258 & 253 & 260 & 244 & 248 & 254 & 237 & 242 & 197 & 243 & 162 \\
\hline $\mathrm{Nb}$ & 0.0 & 0.9 & 0.1 & 0.2 & 0.9 & 0.2 & 1.3 & 1.1 & 0.0 & 0.3 & 0.1 & 0.6 & 0.0 & 0.0 & 0.6 \\
\hline $\mathrm{Ni}$ & 12 & 9 & 12 & 12 & 9 & 11 & 9 & 9 & 13 & 12 & 11 & 6 & 10 & 6 & 8 \\
\hline $\mathrm{Cr}$ & 27 & 12 & 29 & 26 & 12 & 66 & 11 & 12 & 97 & 63 & 20 & 0 & 10 & 19 & 18 \\
\hline $\mathrm{Zn}$ & 58 & 86 & 74 & 16 & 86 & 66 & 82 & 93 & 73 & 72 & 66 & 79 & 79 & 63 & 109 \\
\hline $\mathrm{Cu}$ & & 34 & & & 34 & & 28 & 27 & & & & 39 & & & \\
\hline \multirow{2}{*}{\multicolumn{16}{|c|}{$\begin{array}{l}\mathrm{Nd} \\
\mathrm{Sm}\end{array}$}} \\
\hline & & & & & & & & & & & & & & & \\
\hline $100 \mathrm{Mg} / \mathrm{Mg}+\mathrm{Fe}(\mathrm{t})$ & 50.2 & 51.1 & 48.8 & 48.9 & 51.5 & 49.8 & 50.8 & 52.7 & 52.4 & 50.7 & 51.0 & 51.1 & 51.9 & 32.8 & 55.5 \\
\hline $\mathrm{Al} 2 \mathrm{O} 3 / \mathrm{TIO} 2$ & 33.6 & 35.0 & 31.8 & 32.2 & 32.5 & 33.9 & 34.6 & 32.0 & 32.1 & 32.4 & 35.0 & 32.4 & 33.9 & 33.4 & 33.6 \\
\hline $\mathrm{CaO} / \mathrm{TiO}$ & 18.8 & 17.9 & 17.2 & 16.0 & 16.2 & 18.1 & 17.3 & 16.1 & 15.8 & 15.8 & 17.7 & 15.2 & 15.5 & 15.7 & 15.0 \\
\hline $\mathrm{CaO} / \mathrm{Al} 2 \mathrm{O} 3$ & 0.56 & 0.51 & 0.54 & 0.50 & 0.50 & 0.53 & 0.50 & 0.50 & 0.49 & 0.49 & 0.51 & 0.47 & 0.46 & 0.47 & 0.44 \\
\hline $\mathrm{Ti} / \mathrm{Zr}$ & 81 & 81 & 85 & 85 & 84 & 80 & 78 & 81 & 80 & 80 & 77 & 84 & 75 & 76 & 67 \\
\hline $\mathrm{Zr} / \mathrm{Y}$ & 1.6 & 2.0 & 1.6 & 1.9 & 2.0 & 0.9 & 1.5 & 1.9 & 1.8 & 1.9 & 1.6 & 2.1 & 1.8 & 1.5 & 6.3 \\
\hline
\end{tabular}


Table 7 (continued).

\begin{tabular}{|c|c|c|c|c|c|c|c|c|c|c|c|}
\hline Core, section & $75 R-1$ & $75 R-2$ & $76 \mathrm{R}-1$ & $76 \mathrm{R}-1$ & $76 \mathrm{R}-1$ & $76 \mathrm{R}-1$ & $77 \mathrm{R}-1$ & $78 R-1$ & $78 R-1$ & $78 \mathrm{R}-1$ & $78 R-1$ \\
\hline Interval & $50-55$ & $69-73$ & $43-46$ & $60-64$ & $64-68$ & $85-87$ & $3-7$ & $71-74$ & $80-85$ & $131-136$ & $132-135$ \\
\hline Depth (MBSF) & 850.50 & 856.56 & 860.10 & 861.00 & 860.52 & 862.20 & 867.57 & 879.00 & 880.20 & 883.00 & 884.50 \\
\hline Sample no. & $S-29$ & 114 & $s-30$ & $s-31$ & 116 & $s-32$ & 117 & 118 & $S-33$ & $S-34$ & 119 \\
\hline Unit & 3 & 3 & 5 & 5 & 5 & 5 & 5 & 5 & 5 & 5 & 5 \\
\hline Lithology & Bas.and & Dacite & R.dacite & Bas.and & Bas.and & R.dacite & Dacite & Andesite & Andesite & Dacite & Dacite \\
\hline
\end{tabular}

Major elements (wt\%)

\begin{tabular}{|c|c|c|c|c|c|c|c|c|c|c|c|}
\hline $\mathrm{SiO} 2$ & 53.26 & 62.67 & 73.24 & 55.28 & 55.05 & 74.04 & 62.39 & 59.02 & 58.41 & 64.87 & 63.06 \\
\hline $\mathrm{TiO} 2$ & 0.53 & 0.54 & 0.41 & 0.56 & 0.57 & 0.40 & 0.52 & 0.50 & 0.51 & 0.49 & 0.51 \\
\hline $\mathrm{Al} 2 \mathrm{O} 3$ & 17.66 & 17.63 & 14.03 & 19.32 & 18.87 & 14.43 & 17.91 & 18.48 & 18.74 & 17.77 & 18.09 \\
\hline $\mathrm{Fe} 2 \mathrm{O} 3$ * & 12.37 & 5.84 & 2.92 & 10.04 & 9.81 & 3.72 & 5.49 & 7.22 & 7.94 & 5.08 & 5.08 \\
\hline $\mathrm{MnO}$ & 0.16 & 0.07 & 0.02 & 0.14 & 0.16 & 0.02 & 0.06 & 0.08 & 0.07 & 0.03 & 0.06 \\
\hline $\mathrm{MgO}$ & 6.47 & 2.00 & 0.87 & 5.36 & 5.35 & 0.79 & 1.34 & 3.41 & 3.96 & 1.75 & 1.45 \\
\hline $\mathrm{CaO}$ & 7.85 & 8.17 & 6.13 & 8.31 & 8.17 & 6.23 & 7.97 & 8.61 & 8.88 & 8.35 & 8.40 \\
\hline $\mathrm{Na} 2 \mathrm{O}$ & 1.80 & 2.50 & 2.23 & 2.04 & 2.19 & 2.31 & 3.30 & 2.72 & 2.30 & 2.45 & 2.68 \\
\hline $\mathrm{K} 2 \mathrm{O}$ & 0.12 & 0.16 & 0.24 & 0.14 & 0.20 & 0.22 & 0.48 & 0.17 & 0.14 & 0.37 & 0.40 \\
\hline P2O5 & 0.02 & 0.09 & 0.06 & 0.02 & 0.04 & 0.04 & 0.06 & 0.10 & 0.04 & 0.17 & 0.18 \\
\hline (LOI) & 2.23 & 0.90 & 1.28 & 3.10 & 2.27 & 1.49 & 0.00 & 0.38 & 1.62 & 0.69 & 0.00 \\
\hline Total & 100.24 & 99.67 & 100.15 & 101.21 & 100.41 & 102.20 & 99.52 & 100.31 & 100.99 & 101.33 & 99.91 \\
\hline
\end{tabular}

Trace elements (ppm

\begin{tabular}{|c|c|c|c|c|c|c|c|c|c|c|c|}
\hline $\mathrm{P}$ & & 368 & & & 136 & & 268 & 422 & & & 661 \\
\hline $\mathrm{Zr}$ & 43.8 & 42.3 & 34.7 & 48.7 & 53.8 & 35.7 & 43.1 & 45.2 & 43.7 & 42.3 & 41.6 \\
\hline Y & 5.9 & 26.0 & 15.4 & 6.9 & 7.3 & 22.4 & 21.1 & 19.2 & 10.3 & 30.6 & 29.2 \\
\hline Ga & & 16.2 & & & 15.7 & & 16.6 & 16.0 & & & 16.2 \\
\hline $\mathrm{Rb}$ & 1.6 & 3.6 & 2.7 & 2.1 & 3.4 & 3.1 & 6.0 & 3.3 & 1.2 & 3.9 & 6.1 \\
\hline $\mathrm{Sr}$ & 155 & 165 & 134 & 166 & 159 & 138 & 173 & 167 & 170 & 162 & 163 \\
\hline $\mathrm{Ba}$ & 33 & 23 & 42 & 2 & 0 & 211 & 41 & 15 & 6 & 43 & 36 \\
\hline V & 186 & 235 & 153 & 247 & 216 & 125 & 338 & 205 & 260 & 233 & 225 \\
\hline $\mathrm{Nb}$ & 0.5 & 0.1 & 1.4 & 0.7 & 0.3 & 1.0 & 0.4 & 0.0 & 0.7 & 1.2 & 0.6 \\
\hline $\mathrm{Ni}$ & 5 & 8 & 0 & 7 & 9 & 4 & 6 & 9 & 5 & 4 & 7 \\
\hline $\mathrm{Cr}$ & 0 & 16 & 0 & 2 & 31 & 2 & 22 & 20 & 8 & 4 & 25 \\
\hline $\mathrm{Zn}$ & 134 & 58 & 41 & 98 & 71 & 78 & 50 & 63 & 80 & 55 & 40 \\
\hline $\mathrm{Cu}$ & 18 & & 26 & 17 & & 31 & & & 28 & 18 & \\
\hline \multirow{2}{*}{\multicolumn{12}{|c|}{$\mathrm{Sm}$}} \\
\hline & & & & & & & & & & & \\
\hline $100 \mathrm{Mg} / \mathrm{Mg}+\mathrm{Fe}(\mathrm{t})$ & 53.5 & 43.0 & 39.6 & 54.0 & 54.6 & 31.9 & 34.9 & 51.0 & 52.3 & 43.1 & 38.6 \\
\hline $\mathrm{Al} 2 \mathrm{O} 3 / \mathrm{TIO} 2$ & 33.3 & 32.6 & 34.2 & 34.5 & 33.1 & 36.1 & 34.4 & 37.0 & 36.7 & 36.3 & 35.5 \\
\hline $\mathrm{CaO} / \mathrm{TiO}_{2}$ & 14.8 & 15.1 & 15.0 & 14.8 & 14.3 & 15.6 & 15.3 & 17.2 & 17.4 & 17.0 & 16.5 \\
\hline $\mathrm{CaO} / \mathrm{Al} 2 \mathrm{O} 3$ & 0.44 & 0.46 & 0.44 & 0.43 & 0.43 & 0.43 & 0.45 & 0.47 & 0.47 & 0.47 & 0.46 \\
\hline $\mathrm{Ti} / \mathrm{Zr}$ & 73 & 77 & 71 & 69 & 64 & 67 & 72 & 66 & 70 & 69 & 7 \\
\hline ZilY & 7.4 & 1.6 & 2.3 & 7.1 & 7.4 & 1.6 & 2.0 & 2.4 & 4.2 & 1.4 & 1.4 \\
\hline
\end{tabular}



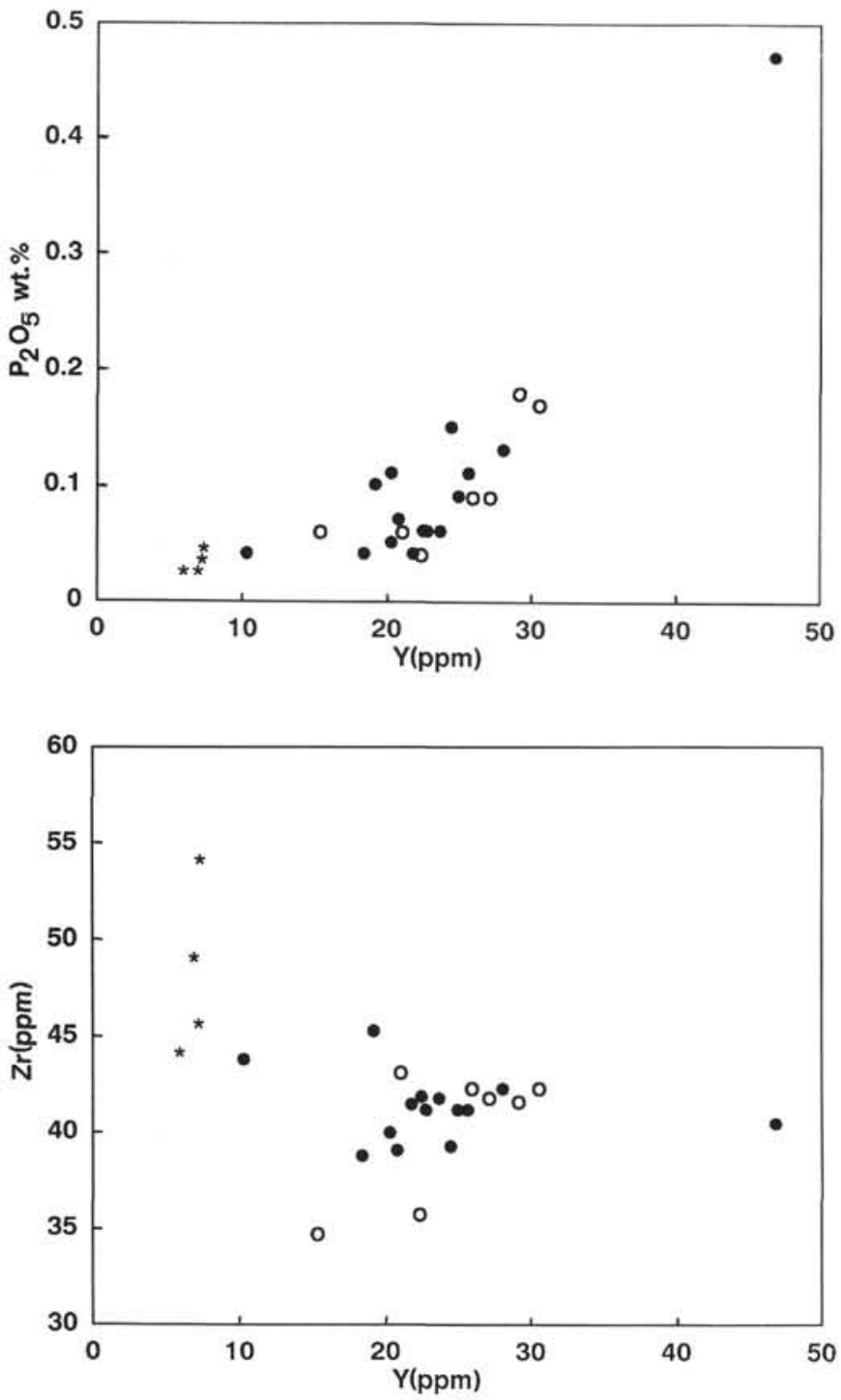

Figure 12. $\mathrm{P}_{2} \mathrm{O}_{5}$ and $\mathrm{Zr}$ plotted vs. $\mathrm{Y}$ for Hole $792 \mathrm{E}$ basement.

acteristic of boninitic volcanism is the nature of enrichments to their source. Figure 18 combines two parameters that distinguish boninites from arc and oceanic basalt compositions. Western Pacific boninites have lower $\mathrm{Ti} / \mathrm{Zr}$ ratios and $\varepsilon_{\mathrm{Nd}}$ compared with MORB and lavas from the active Izu-Mariana arcs. These features have been ascribed to enrichment in light $\mathrm{REE}$ and $\mathrm{Zr}$ relative to middle/heavy $\mathrm{REE}, \mathrm{Ti}$, and Y (Sun and Nesbitt, 1978; Hickey and Frey, 1982; Cameron, 1985;

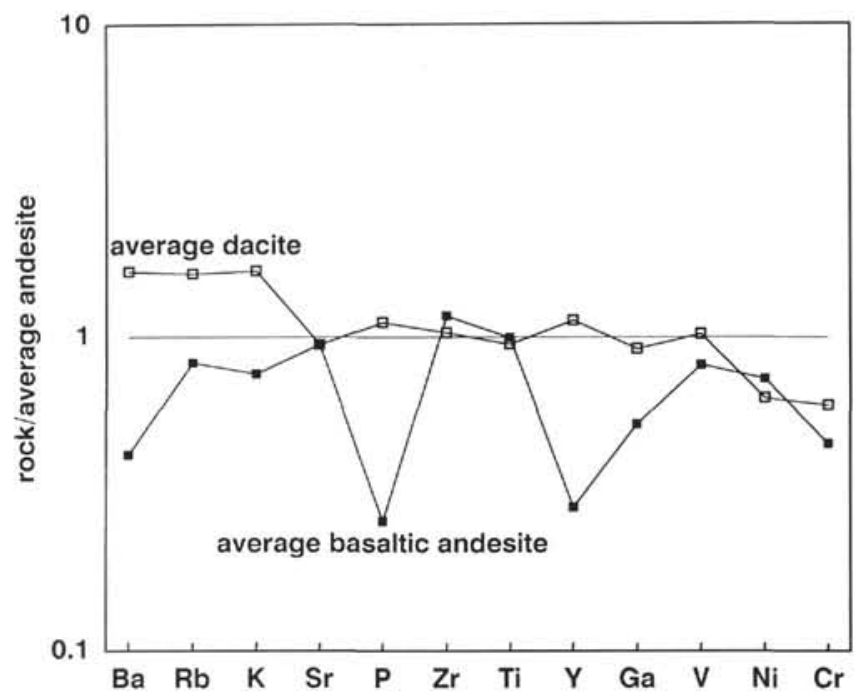

Figure 13. Average trace element compositions for average dacite (Unit 5) and average basaltic andesite (Unit 5) normalized to average andesite (Unit 1).

Taylor and Nesbitt, 1988). Samples from the Hole 793B basement are intermediate between arc and boninite in terms of both parameters in Figure 18.

It is notable that the Site 793 lavas are geochemically very similar to Site 458 bronzite andesites from the Mariana forearc basin. Lavas from both these sites are intermediate in composition between respective active arc and outer-arc high lavas (e.g., Fig. 18). This is taken to reflect their similar tectonic setting (forearc basin) and age (Hussong, Uyeda, et al., 1981; Taylor and Mitchell, this volume). Geochemical transects across the Izu-Bonin and Mariana arcs demonstrate a shallower source for the basin volcanics relative to the arc volcanics (Taylor et al., 1992), and a parallel mantle evolution along the length of the Izu-Mariana system.

\section{CONCLUSIONS}

Drilling during Leg 126 determined the composition and nature of lavas within the Izu-Bonin forearc basin. Basement at the center of the basin (Site 793) consists of Oligocene synrift volcanics. These are two-pyroxene basaltic andesites, with HFSE contents lower than the active Izu arc. On the western edge of the forearc basin (Site 792), lavas were recovered from a basement promontory, postulated as a relict arc volcano. The volcanics from this site are massive flows and breccias of two-pyroxene andesite and dacite with calc-alkaline affinities.

At Site 793, basement lavas show anomalous behavior of P, Y, and REE relative to HFS elements. These can be attributed to posteruptive fluid-rock interaction, which redistributed P, Y, and REE within the basement system. Similar discrepancies are found in the Site 792 lavas,

Table 8. Nd, $\mathrm{Sr}$, and $\mathrm{Pb}$ isotopic results from Hole 793B.

\begin{tabular}{|c|c|c|c|c|c|c|c|c|c|c|c|c|c|c|c|c|}
\hline $\begin{array}{l}\text { Core } \\
\text { Interval } \\
\text { Sample number } \\
\text { Lithology }\end{array}$ & $\begin{array}{c}\text { IR-2 } \\
61-65 \\
007 \\
\text { diabase }\end{array}$ & $\begin{array}{c}86 R-1 \\
128-131 \\
020 \\
\text { cpx-bas }\end{array}$ & $\begin{array}{c}88 \mathrm{R}-1 \\
65-68 \\
\text { S-8 } \\
\text { aph bas }\end{array}$ & $\begin{array}{c}92 R-2 \\
70-74 \\
030 \\
\text { cpx-pill }\end{array}$ & $\begin{array}{c}92 \mathrm{R}-3 \\
25-28 \\
\text { S-10 } \\
\text { cpx-pill }\end{array}$ & $\begin{array}{c}97 \mathrm{R}-1 \\
124-127 \\
\text { S-13 } \\
\text { cpo mas }\end{array}$ & $\begin{array}{l}\text { 99R-1 } \\
54-55 \\
\text { S-14 } \\
\text { cpx-pill }\end{array}$ & $\begin{array}{c}104 \mathrm{R}-1 \\
65-70 \\
053 \\
\text { cpo mas }\end{array}$ & $\begin{array}{c}104 \mathrm{R}-2 \\
49-54 \\
\mathrm{~S}-18 \\
\text { cpc mas }\end{array}$ & $\begin{array}{c}104 \mathrm{R}-2 \\
69-75 \\
055 \\
\text { cpc mes }\end{array}$ & $\begin{array}{c}105 R-1 \\
127-131 \\
\text { S-17 } \\
\text { cpx-pill }\end{array}$ & $\begin{array}{c}110 R-1 \\
1-3 \\
S-19 \\
c-s p o t\end{array}$ & $\begin{array}{c}111 \mathrm{R}-1 \\
110-115 \\
079 \\
\text { cpc mas }\end{array}$ & $\begin{array}{c}112 \mathrm{R}-1 \\
59-63 \\
\text { S-20 } \\
\text { cpc mas }\end{array}$ & $\begin{array}{c}113 R-3 \\
137-141 \\
S-21 \\
\text { aph bas }\end{array}$ & $\begin{array}{c}113 R-4 \\
35-41 \\
088 \\
\text { aph bas }\end{array}$ \\
\hline $\begin{array}{l}\mathrm{Sr} \\
\mathrm{Nd} \\
\mathrm{Sm}\end{array}$ & 116 & 129 & $\begin{array}{l}156 \\
4.64 \\
1.48\end{array}$ & 135 & $\begin{array}{l}163 \\
5.92 \\
1.73\end{array}$ & $\begin{array}{l}140 \\
2.46 \\
0.84\end{array}$ & $\begin{array}{l}140 \\
3.69 \\
1.21\end{array}$ & 161 & $\begin{array}{l}178 \\
5.61 \\
1.78\end{array}$ & 201 & $\begin{array}{l}129 \\
2.77 \\
0.97\end{array}$ & $\begin{array}{l}147 \\
3.46 \\
1.06\end{array}$ & 152 & $\begin{array}{l}187 \\
3.28 \\
1.04\end{array}$ & $\begin{array}{l}196 \\
7.36 \\
2.32\end{array}$ & 195 \\
\hline $\begin{array}{l}{ }_{87 / 866}^{143 / 144} \mathrm{Nd} \\
\varepsilon_{\mathrm{Nd}}\end{array}$ & & & $\begin{array}{c}0.51296 \\
0.70377 \\
6.4\end{array}$ & & $\begin{array}{c}0.51296 \\
0.70410 \\
6.4\end{array}$ & $\begin{array}{c}0.51296 \\
0.70384 \\
6.2\end{array}$ & $\begin{array}{c}0.51295 \\
0.70397 \\
6.1\end{array}$ & & $\begin{array}{c}0.51299 \\
0.70383 \\
6.8\end{array}$ & & $\begin{array}{c}0.51303 \\
0.70394 \\
5.6\end{array}$ & $\begin{array}{c}0.61296 \\
0.70383 \\
6.3\end{array}$ & & $\begin{array}{c}0.51296 \\
0.70439 \\
6.2\end{array}$ & $\begin{array}{c}0.51297 \\
0.70389 \\
6.5\end{array}$ & \\
\hline $\begin{array}{l}{ }_{206 / 204}^{200} \mathrm{~Pb} \\
{ }_{207204} \mathrm{~Pb} \\
{ }_{206 / 204} \mathrm{~Pb}\end{array}$ & $\begin{array}{l}18.35 \\
15.56 \\
38.33\end{array}$ & $\begin{array}{l}18.20 \\
15.49 \\
37.93\end{array}$ & & $\begin{array}{l}18.18 \\
15.46 \\
37.81\end{array}$ & & & & $\begin{array}{l}18.22 \\
15.48 \\
37.91\end{array}$ & & $\begin{array}{l}18.21 \\
15.47 \\
37.87\end{array}$ & & & $\begin{array}{l}18.16 \\
15.47 \\
37.84\end{array}$ & & & $\begin{array}{l}18.17 \\
15.44 \\
37.80\end{array}$ \\
\hline
\end{tabular}




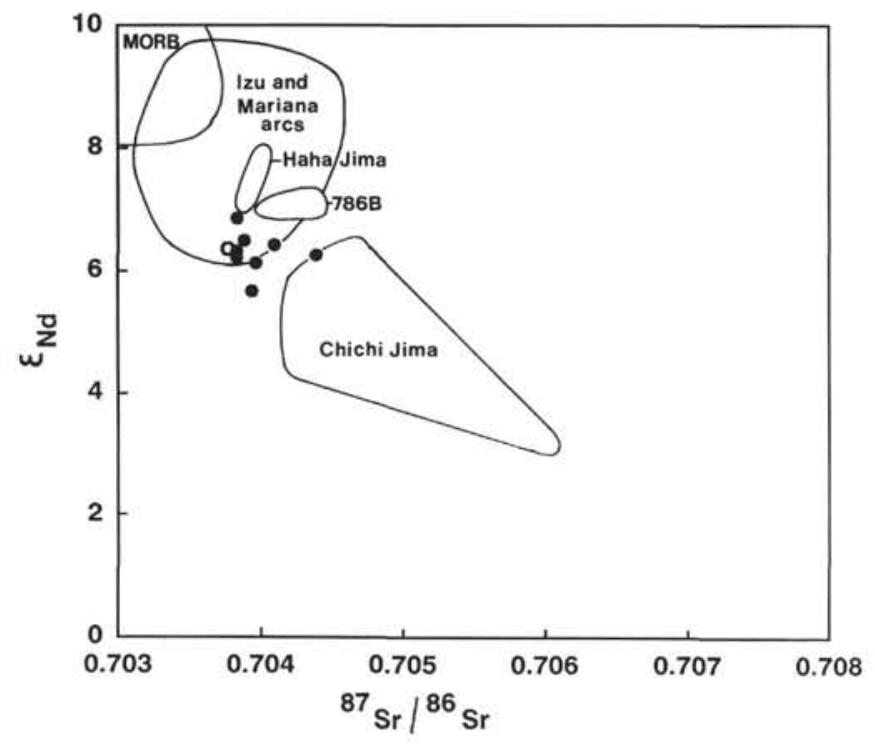

Figure 14. $\varepsilon_{\mathrm{Nd}}$ plotted vs. ${ }^{87} \mathrm{Sr} /{ }^{86} \mathrm{Sr}$ for Hole $793 \mathrm{~B}$ basement. Fields for Izu and Mariana arcs from Woodhead (1989) and Nohda and Wasserburg (1981); ODP Leg 125 fields for Hole 786B from Pearce et al. (1992); and Chichi Jima and Haha Jima from Taylor, Nesbitt, and Vidal (unpubl. data).

with more extreme element redistribution. Groundmass changes and fluid movements have produced $\mathrm{SiO}_{2}$-rich and $\mathrm{SiO}_{2}$-poor meta-andesites with characteristic concentration and dilution of HFS elements.

Age relationships of basement lavas from the two forearc Sites (792 and 793) indicate that lavas from the forearc basin are younger than the Oligocene arc at Site 792. This demonstrates that the current Izu-Bonin forearc crust was in a forearc location during Oligocene rifting and experienced synrift volcanism.

Trace element and isotopic evidence indicates that the Hole 793B basement volcanics are transitional between arc-tholeiite and boninite chemistry. This suggests that their intermediate location between the outer-arc high (boninitic) and the active arc (arc-tholeiite) can be related to a gradual change in melting environment toward the trench.

\section{ACKNOWLEDGMENTS}

This work was supported by Natural Environment Research Council Grant No. GST/02/416 (RNT and RWN). RNT would like to thank all Leg 126 participants for a good cruise, and I. Chaplin for rock preparation logistics. The reviewers are thanked for their helpful and constructive comments.

\section{REFERENCES}

Bienvenu, P., Bougault, H., Joron, J. L., Treuil, M., and Dmitriev, L., 1990. MORB alteration: rare-earth element/non-rare-earth hygromagmaphile element fractionation. Chem. Geol., 82:1-14.

Bloomer, S. H., and Hawkins, J. W., 1987. Petrology and geochemistry of boninite series volcanic rocks from the Mariana trench. Contrib. Mineral. Petrol., 97:361-377.

Cameron, W. E., 1985. Petrology and origin of primitive lavas from the Troodos ophiolite, Cyprus. Contrib. Mineral. Petrol., 89:239-255.

Croudace, I. W., and Gilligan, J., 1990. Versatile and accurate trace element determinations in iron-rich and other geological samples using $\mathrm{X}$-ray fluorescence analysis. X-ray Spectrom., 19:117-123.

Croudace, I. W., and Marshall, S., 1991 Determination of rare-earth elements and yttrium in nine geochemical reference samples using a novel group separation procedure involving mixed acid elution ion-exchange chromatography. Geostand. Newsl., 15:139-144.

Dietrich, V., Emmermann, R., Oberhänsli, R., and Puchelt, H., 1978. Geochemistry of basaltic and gabbroic rocks from the west Mariana basin and the Marina trench. Earth Planet. Sci. Lett., 39:127-144.

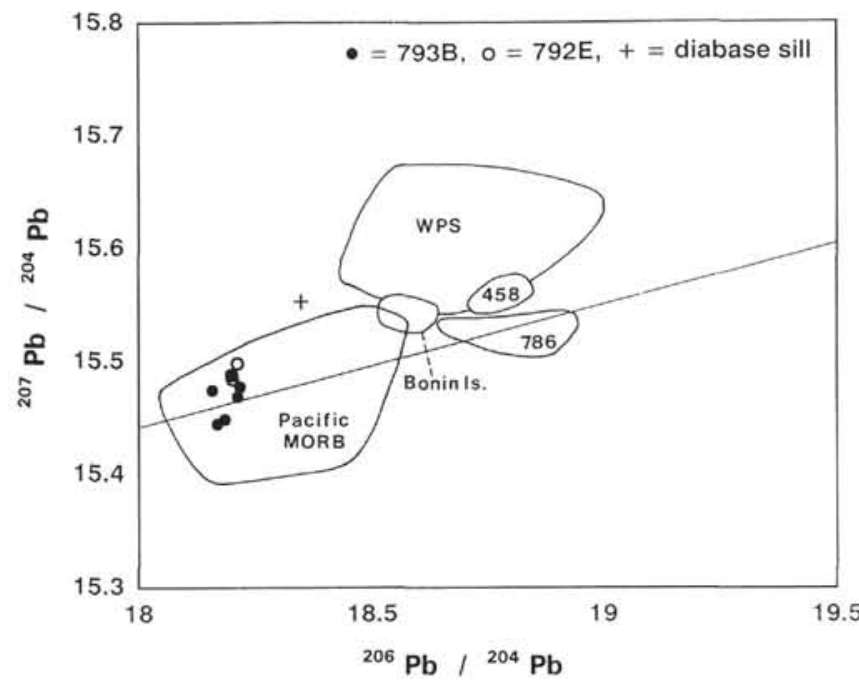

Figure 15. ${ }^{207} \mathrm{~Pb} /{ }^{204} \mathrm{~Pb}-{ }^{206} \mathrm{~Pb} /{ }^{204} \mathrm{~Pb}$ for Leg 126 igneous rocks. Fields are shown for Pacific MORB (Tatsumoto, 1978; Vidal and Clauer, 1981); western Pacific sediment (WPS; Sun, 1980; Meijer, 1976; Woodhead and Fraser, 1985); Bonin islands (Taylor et al., unpubl. data); DSDP Site 458 (Hickey-Vargas, 1989); and ODP Site 786 (Pearce et al., 1992).

Dobson, P. F., and Tilton, G. R., 1989. Th, U, and Pb systematics of boninite series volcanic rocks from Chichi-Jima, Bonin Islands, Japan. In Crawford, A. J. (Ed.), Boninites and Related Rocks: London (Unwin Hyman), 396-415.

Egeberg, P. K., and Leg 126 Shipboard Scientific Party, 1990. Unusual composition of pore-waters found in the Izu-Bonin fore-arc sedimentary basin. Nature, 344:215-218.

Fryer, P., Pearce, J. A., Stokking, L. B., et al., 1990. Proc. ODP, Init. Repts., 125: College Station, TX (Ocean Drilling Program).

Henderson, P., 1982. Inorganic Geochemistry: Oxford (Pergamon Press).

Hickey, R. L., and Frey, F. A., 1982, Geochemical characteristics of boninite series volcanics: implications for their source. Geochim. Cosmochim. Acta, 46:2099-2115.

Hickey-Vargas, R. L., 1989. Boninites and tholeites from DSDP Site 458, Mariana forearc. In Crawford, A. J. (Ed.), Boninites and Related Rocks: London (Unwin Hyman), 339-356.

Honza, E., and Tamaki, K., 1985. The Bonin Arc. In Nairn, A.E.M., Stehli, F. G., and Uyeda, S. (Eds.), The Ocean Basins and Margins (Vol. 7): New York (Plenum), 459-502.

Kay, R. W., and Gast, P. W., 1973. The rare-earth content and origin of alkali-rich basalts. J. Geol., 81:653-682.

Leg 126 Shipboard Scientific Party, 1989a. Arc volcanism and rifting. Nature, $342: 18-20$.

, 1989b. ODP Leg 126 drills the Izu-Bonin Arc. Geotimes, 34:36-38.

Meijer, A., 1976. $\mathrm{Pb}$ and $\mathrm{Sr}$ isotopic data bearing on the origin of volcanic rocks from the Mariana island-arc system. Geol. Soc. Am. Bull., 87:358-369.

Nohoda, S., and Wasserburg, G. J., 1981. Nd and Sr isotopic study of volcanic rocks from Japan. Earth Planet. Sci. Lett., 52:264-276.

Pearce, J. A., Thirlwall, M., Ingram, G., Murton, B. J., Arculus, R. J., and Van Der Lann, S. R., 1992. Isotopic evidence for the origin and evolution of the Izu-Ogasawara forearc at Sites 782 and 786. In Fryer, P., Pearce, J. A. Stokking, L. B., et al., Proc. ODP, Sci. Results, 125: College Station, TX (Ocean Drilling Program).

Saunders, A. D., Tarney, J., Marsh, N. G., and Wood, D. A., 1980. Ophiolites as ocean crust or marginal basin crust: a geochemical approach. In Panayiotou, A. (Ed.), Ophiolites: Geol. Surv. Dept Cyprus, 193-204.

Shipboard Scientific Party, 1990. Sites 788/789. In Taylor, B., Fujioka, K., et al., Proc. ODP, Init. Repts., 126: College Station, TX (Ocean Drilling Program), 97-126.

Sun, S. S., 1980. Lead isotopic study of young volcanic rocks from mid-ocean ridges, ocean islands and island arcs. Philos. Trans. R. Soc. London, Ser. A, 297:409-445.

Sun, S.-S., and Nesbitt, R. W., 1978. Geochemical regularities and genetic significance of ophiolitic basalts. Geology, 6:689-693. 


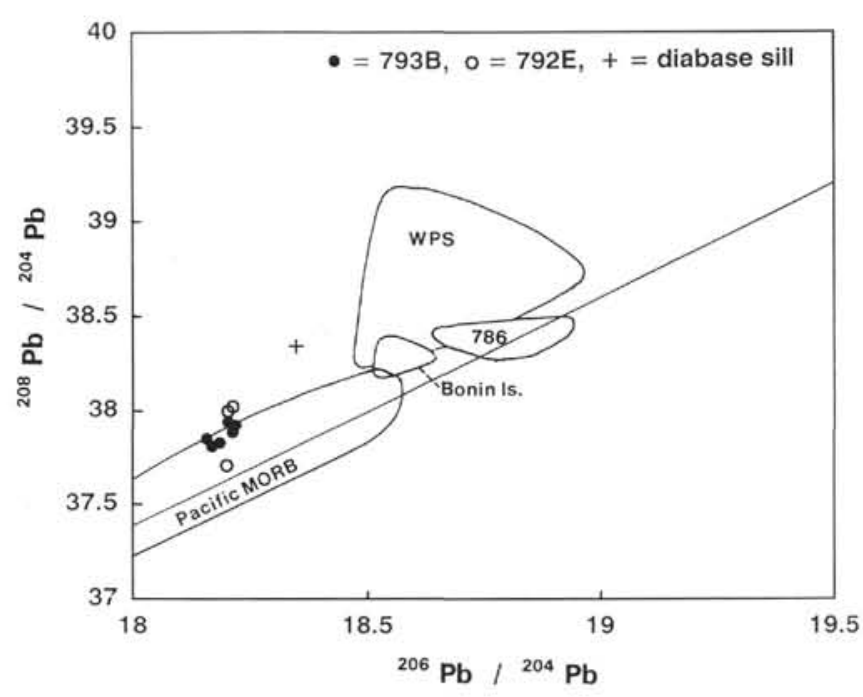

Figure 16. ${ }^{208} \mathrm{~Pb} /{ }^{204} \mathrm{~Pb}-{ }^{206} \mathrm{~Pb} /{ }^{204} \mathrm{~Pb}$ for Leg 126 igneous rocks. Fields as for Figure 15 .

Tatsumoto, M., 1978. Isotopic composition of lead in oceanic basalt and its implication to mantle evolution. Earth Planet. Sci. Lett., 38:63-87.

Taylor, B., Fujioka, K., et al., 1990. Proc. ODP, Init. Repts., 126: College Station, TX (Ocean Drilling Program).

Taylor, B., Moore, G., Klaus, A., Systrom, M., Cooper, P., and MacKay, M., 1990. Multichannel seismic survey of the central Izu-Bonin Arc. In Taylor, B., Fujioka, K., et al., Proc. ODP, Init. Repts., 126: College Station, TX (Ocean Drilling Program), 51-60.

Taylor, R. N., Murton, B. J., and Nesbitt, R. W., 1992. Chemical transects across intra-oceanic arcs: implications for the tectonic setting of ophiolites. Spec. Publ., Geol. Soc. London, 60:117-132.

Taylor, R. N., and Nesbitt, R. W., 1988. Light rare-earth enrichment of supra-subduction-zone mantle: evidence from the Troodos ophiolite, Cyprus. Geology, 16:448-451.

Umino, S., 1986. Geological and petrological study of boninites and related rocks from Chichi Jima, Bonin Islands [Ph.D. thesis]. Univ. Tokyo.

Vidal, P., and Clauer, N., 1981. Pb and Sr isotopic systematics of some basalts and sulphides from the East Pacific Rise at $21^{\circ} \mathrm{N}$ (Project RITA). Earth Planet. Sci. Lett., 55:237-246.

Woodhead, J. D., 1989. Geochemistry of the Mariana arc (western Pacific): source composition and processes. Chem. Geol., 76:1-24.

Woodhead, J. D., and Fraser, D. G., 1985. Pb, Sr, and ${ }^{10} \mathrm{Be}$ isotopic studies of volcanic rocks from the Northern Mariana Islands: implications for magma genesis and crustal recycling in the western Pacific. Geochim. Cosmochim. Acta, 49:1925-1930.

Date of initial receipt: 26 December 1990

Date of acceptance: 30 September 1991

Ms 126B-146

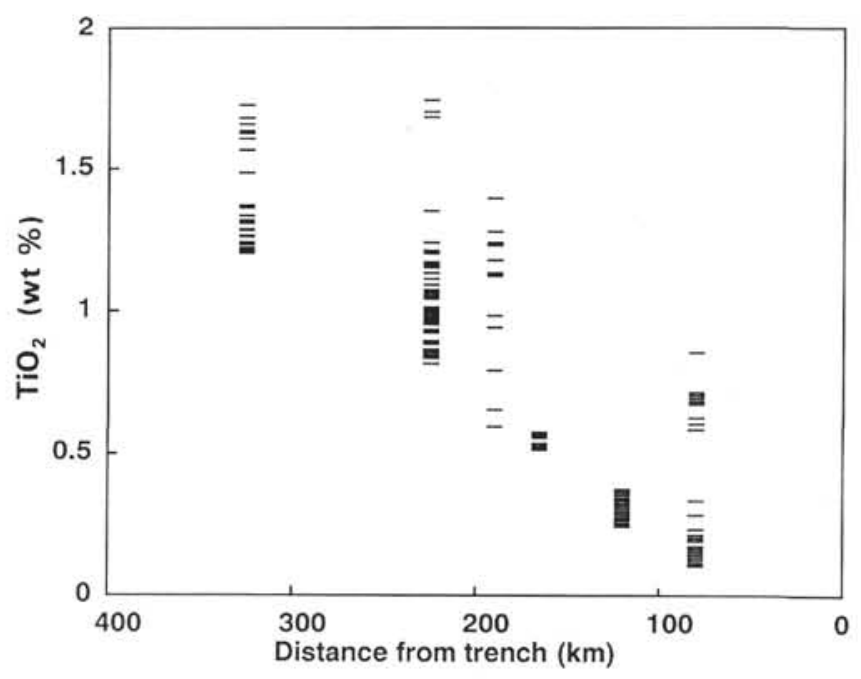

Figure 17. $\mathrm{TiO}_{2}$ content of lavas from the Izu-Bonin arc-trench system plotted vs. distance from the trench. Details of samples used and locations are given in Taylor et al. (1992).

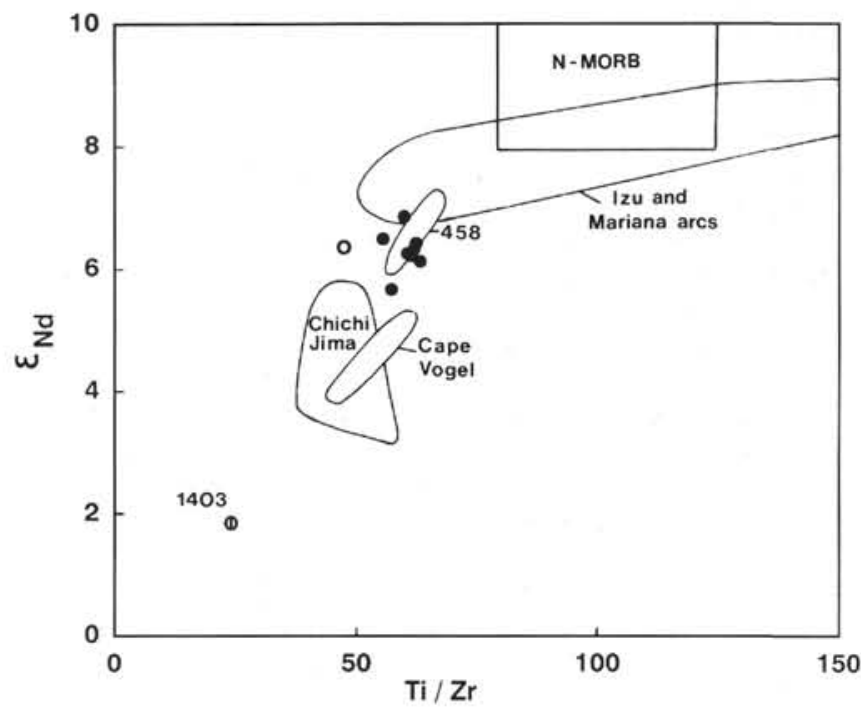

Figure $18 . \mathrm{Ti} / \mathrm{Zr}$ plotted vs. $\varepsilon_{\mathrm{Nd}}$ for Hole 793B basement. Data fields for Izu and Mariana arcs from Nohda and Wasserburg (1981) and Woodhead (1989). Site 458, Site 1403, and Cape Vogel from Hickey and Frey (1982). Chichi Jima from Taylor, Nesbitt, and Vidal (unpubl. data). 


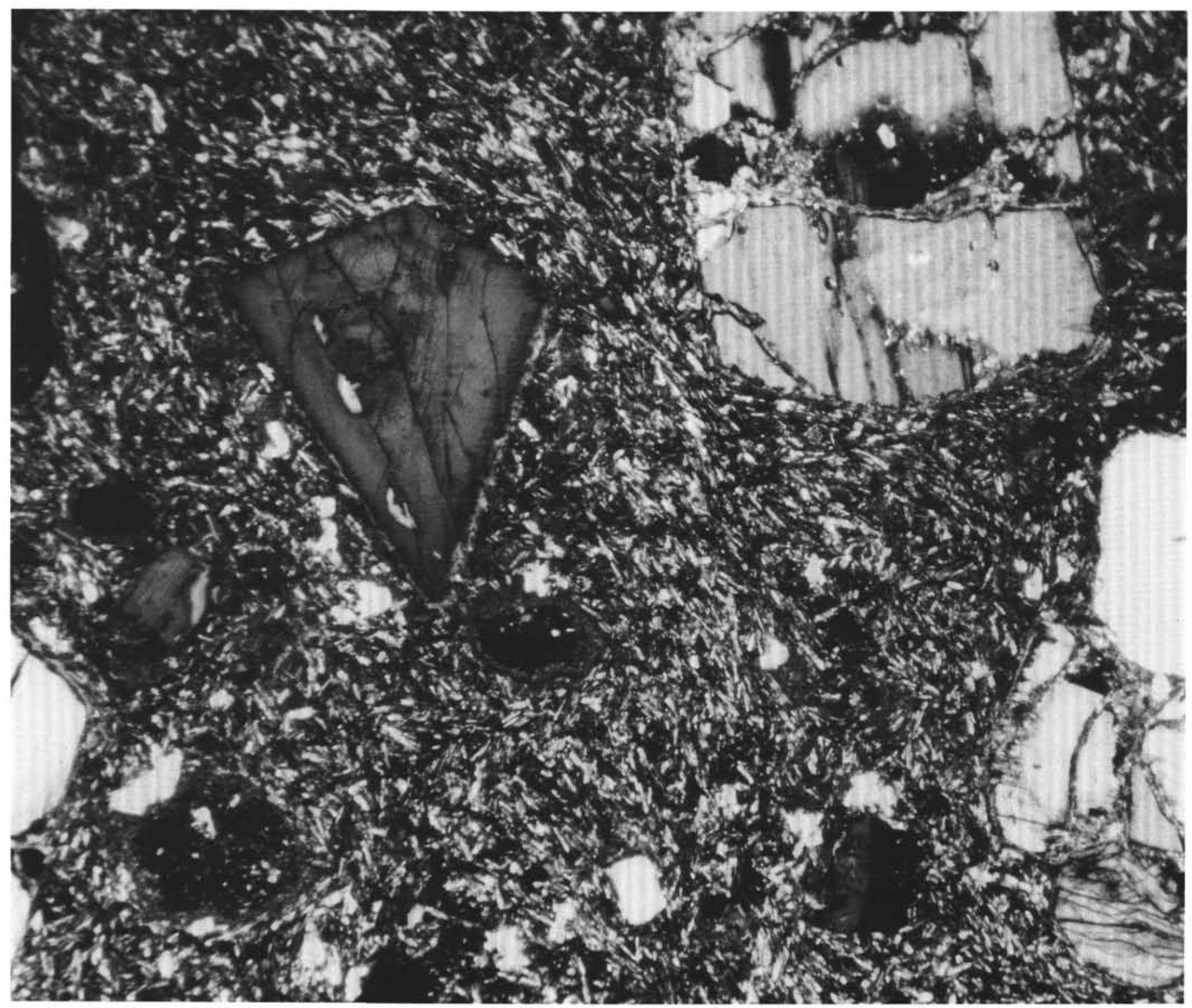

Plate 1. Photomicrograph from Sample 126-793B-110R-1, $1 \mathrm{~cm}$ (S-19); width of field = $7 \mathrm{~mm}$; cross-polarized light; basaltic andesite massive flow (Unit 13). Euhedral phenocrysts of orthopyroxene (upper right) and clinopyroxene (center left) set in a groundmass of altered glass and plagioclase microlites. Vesicles (dark patches) are coated with smectite. 


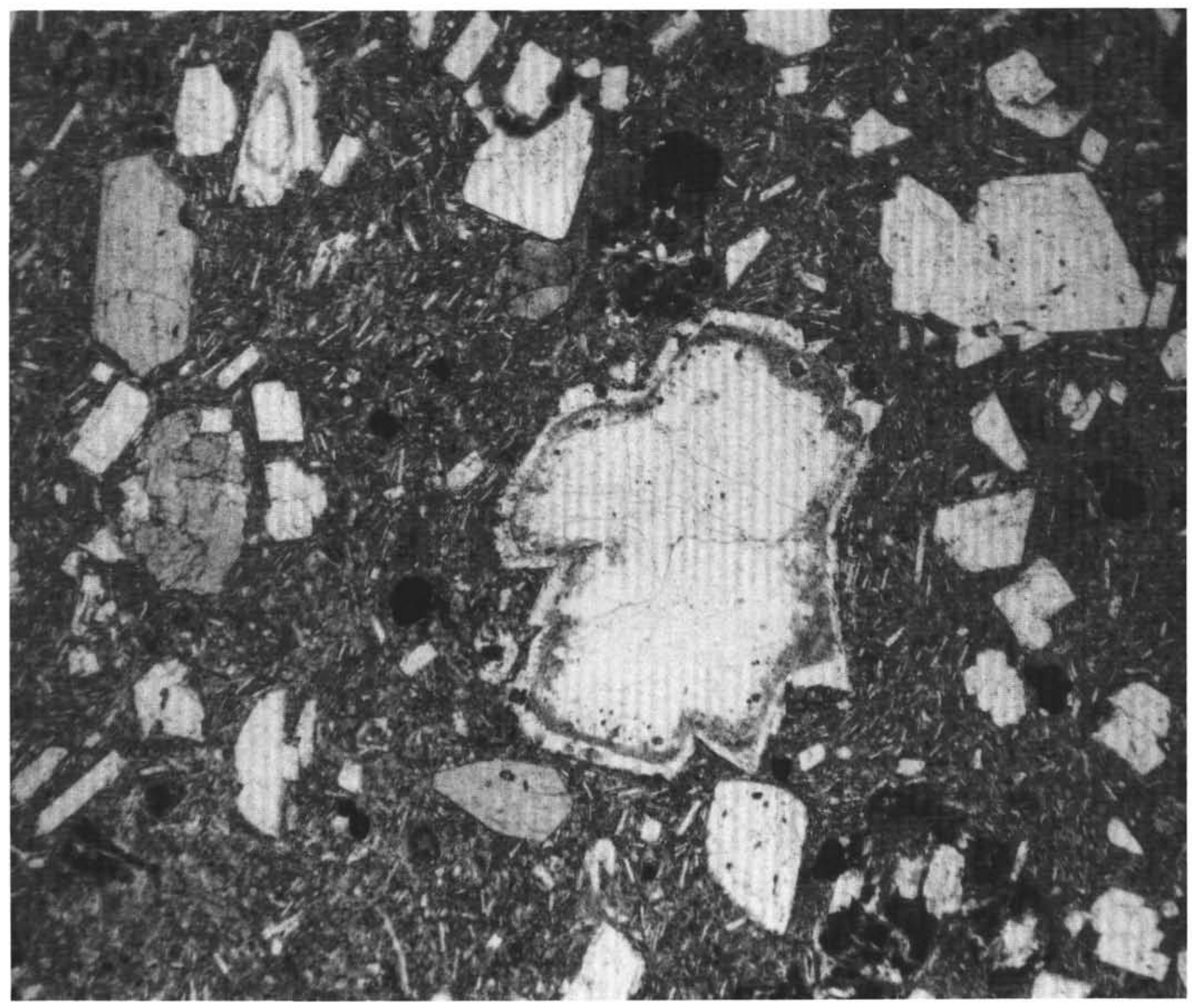

Plate 2. Photomicrograph from Sample 126-792E-71R-3, $51 \mathrm{~cm}$; width of field $=6 \mathrm{~mm}$; plane polarized light; typical texture in a two-pyroxene andesite from Hole 792E basement. Large plagioclase with fluid/melt inclusion zone occupies center of field. Other phenocrysts include clinopyroxene (center left) and orthopyroxene pseudomorphs (lower right). 